\title{
Local theory for ions in binary liquid mixtures
}

\author{
Markus Bier, ${ }^{1, *}$ Andrea Gambassi, ${ }^{2}$ and S. Dietrich ${ }^{1}$ \\ 1 Max-Planck-Institut für Intelligente Systeme, Heisenbergstr. 3, 70569 Stuttgart, \\ Germany, and Institut für Theoretische und Angewandte Physik, \\ Universität Stuttgart, Pfaffenwaldring 57, 70569 Stuttgart, Germany \\ ${ }^{2}$ SISSA - International School for Advanced Studies and INFN, via Bonomea 265, 34136 Trieste, Italy
}

(Dated: 6 July, 2012)

\begin{abstract}
The influence of ions on the bulk phase behavior of binary liquid mixtures acting as their solvents and on the corresponding interfacial structures close to a planar wall is investigated by means of density functional theory based on local descriptions of the effective interactions between ions and their solvents. The bilinear coupling approximation (BCA), which has been used in numerous previous related investigations, is compared with a novel local density approximation (LDA) for the ion-solvent interactions. It turns out that within BCA the bulk phase diagrams, the two-point correlation functions, and critical adsorption exhibit qualitative features which are not compatible with the available experimental data. These discrepancies do not occur within the proposed LDA. Further experimental investigations are suggested which assess the reliability of the proposed LDA. This approach allows one to obtain a consistent and rather general understanding of the effects of ions on solvent properties. From our analysis we infer in particular that there can be an experimentally detectable influence of ions on binary liquid mixtures due to steric effects but not due to charge effects.
\end{abstract}

\section{INTRODUCTION}

Ion-solvent mixtures play a central role for various important soft matter systems such as colloidal suspensions, polymer solutions, biochemical reactions, and electrochemical cells. For most of these systems the presence of an appropriate amount of ions is crucial for their functioning. Therefore the general question arises of how and to what extent ions alter the properties of a solvent. More than a century ago the considerations by Arrhenius concerning the colligative properties of ionic solutions [1] seemed to imply that the interactions of ions with their fluid environment have only a mild effect in the sense that adding salt merely modifies the composition of the ionsolvent mixture. A few decades later, after more accurate measurements had been carried out, this simple picture was questioned. Bjerrum, Debye, and Hückel pointed out that electrostatically induced ion-ion correlations are expected to influence the ion distribution and the equation of state of electrolyte solutions [2, 3]. Since then, the Debye-Hückel theory has been widely used, e.g., in plasma physics and as an ingredient of the DLVO theory (named after Derjaguin, Landau, Verwey, and Overbeek) of colloidal suspensions [4]. Within Debye-Hückel theory the solvent is considered to be a uniform dielectric continuum which influences the Coulomb interaction between the ions via a certain permittivity but which is otherwise inert. Recently the issue of the mutual influence of ions and the solvent has been taken up again, studying the solubility of ions and the double-layer structure in a near-critical solvent [5-9], possible salt-induced changes of the solvent structure [10-17], and effects of the inho-

*Electronic address: bier@is.mpg.de mogeneities of the permittivity close to interfaces [18-21].

These investigations require a model for the solvent at least on the mesoscopic scale as well as a description of the ion-solvent interaction. To this end one can split the pair potential between the species into the long-ranged electrostatic monopole-monopole contribution and into the remaining contributions of shorter range, which we shall refer to as chemical contributions. In the vast majority of the theoretical studies of dilute electrolyte solutions, ions are described as point-like particles whose chemical contributions to the interactions with the solvent are modeled locally within the so-called bilinear coupling approximation (BCA). This amounts to a local density approximation for the chemical contribution to the excess free energy which is bilinear in the particle number densities [5-8, 10-12, 18, 19, 21]. Within the approaches of Refs. [9] and [20] the ion size is accounted for by means of hard-core exclusion and solvation is modeled by nonlocal interactions within the so-called random-phase approximation (RPA) for density functional theory (DFT). In fact, the BCA can be considered as the local version of the RPA, which is expected to be reliable only for interaction energies small compared with the thermal energy [22]. However, the ion-solvent interaction is typically of the order of some tens of the thermal energy [23, 24]. Therefore the application of the BCA or of the RPA to ion-solvent mixtures is questionable [25].

In the following we show that for realistic values of the parameters the BCA and the RPA indeed lead to unphysical results. Since non-local models are notoriously complicated it seems worthwhile to investigate the possibility of local descriptions of ion-solvent interactions. In order to demonstrate that valuable improvements within the class of local models are possible, we propose an alternative local density approximation (LDA) the predictions of which are in qualitative agreement with experimental re- 
sults and which does not lead to the artifacts introduced by the BCA. Without presenting the details which will be expounded below, this LDA has already been applied successfully in a recent study of the effective interaction between two planar substrates in contact with a nearcritical binary liquid mixture and in the presence of salt [26]. As far as the phase behavior, the bulk structure, and the asymptotic interfacial structure of the solvent are concerned, our results are equivalent to replacing the bare interaction energies used within the BCA by effective ones which saturate for large values of the bare interaction energies. Within this LDA, and in contrast to the $\mathrm{BCA}$, more realistic estimates of the magnitude of saltinduced effects can be obtained, which are expected to be important for interpreting and designing experiments and for considering applications.

In the following, after introducing the model and the LDA in Sec. II and in Appendix A, bulk systems are discussed in Sec. III, where we focus on the phase diagram and on the structure of the correlation functions. Similarities and differences between the proposed LDA and the BCA are highlighted and compared with the available experimental data. In Sec. IV interfacial structures as well as critical adsorption in semi-infinite planar systems are discussed. For critical adsorption important qualitative differences between the LDA and the BCA are revealed, with the predictions of the former being in agreement with the corresponding qualitative experimental findings, whereas those of the latter are not. We propose further experimental investigations which are expected to discriminate more sharply between the LDA and the BCA than the presently available data do. In Sec. V an application of the proposed LDA to colloidal interactions in near-critical electrolyte solutions is discussed and compared with alternative approaches described in the literature. Finally, in Sec. VI we draw our conclusions and provide a summary.

\section{MODEL}

\section{A. Definition}

We consider a three-dimensional $(d=3)$ container $\widetilde{\mathcal{V}} \subseteq \mathbb{R}^{3}$ filled with an incompressible binary liquid mixture acting as a solvent for cations $(+)$ and anions $(-)$. All solvent particles are assumed to be of equal size with non-vanishing volume $\widetilde{a}^{3}$ whereas the ions are considered to be point-like; hence ions do not contribute to the total packing fraction (see also Appendix A). The set of dimensionless positions $\mathbf{r}=(x, y, z):=\widetilde{\mathbf{r}} / \widetilde{a}$ for $\widetilde{\mathbf{r}} \in \widetilde{\mathcal{V}}$ is defined as $\mathcal{V}$. At $\mathbf{r} \in \mathcal{V}$ the number densities of the solvent components $A$ and $B$ are given by $\widetilde{\varrho}_{A}(\mathbf{r})=\phi(\mathbf{r}) \widetilde{a}^{-3}$ and $\widetilde{\varrho}_{B}(\mathbf{r})=(1-\phi(\mathbf{r})) \widetilde{a}^{-3}$, respectively, with $0 \leq \phi \leq 1$, whereas the number densities of the cations and anions are given by $\widetilde{\varrho}_{+}(\mathbf{r})=\varrho_{+}(\mathbf{r}) \widetilde{a}^{-3}$ and $\widetilde{\varrho}_{-}(\mathbf{r})=\varrho_{-}(\mathbf{r}) \widetilde{a}^{-3}$, respectively. The walls $\partial \mathcal{V}$ of the container carry a surface charge density $\sigma(\mathbf{r}) e \widetilde{a}^{-2}$ at $\mathbf{r} \in \partial \mathcal{V}$, where $e$ is the (positive) elementary charge. The influence of the walls onto the solvent due to short-ranged chemical effects is captured by surface fields localized at the walls. At $\mathbf{r} \in \partial \mathcal{V}$, the dimensionless volume fraction $\phi(\mathbf{r})$ of $A$ particles couples linearly to surface fields $h(\mathbf{r})$, where $h>0(<0)$ leads to a preferential adsorption of solvent component $A(B)$. The equilibrium profiles $\phi$, $\varrho_{+}$, and $\varrho_{-}$minimize the approximate grand potential density functional $k_{B} T \Omega\left[\phi, \varrho_{ \pm}\right]$,

$$
\begin{aligned}
\Omega\left[\phi, \varrho_{ \pm}\right]= & \int_{\mathcal{V}} \mathrm{d}^{3} r\left\{\omega_{\text {sol }}(\phi(\mathbf{r}))+\frac{\chi(T)}{6}(\nabla \phi(\mathbf{r}))^{2}\right. \\
& +\sum_{i= \pm}\left[\omega_{\text {ion }}^{(i)}\left(\varrho_{i}(\mathbf{r})\right)+\varrho_{i}(\mathbf{r}) V_{i}(\phi(\mathbf{r}))\right] \\
& \left.+\frac{2 \pi \ell_{B}}{\varepsilon(\phi(\mathbf{r}))} \mathbf{D}\left(\mathbf{r},\left[\varrho_{ \pm}\right]\right)^{2}\right\}-\int_{\partial \mathcal{V}} \mathrm{d}^{2} r h(\mathbf{r}) \phi(\mathbf{r})
\end{aligned}
$$

with $\omega_{\text {sol }}(\phi)=\phi\left(\ln \phi-\mu_{\phi}\right)+(1-\phi) \ln (1-\phi)+\chi(T) \phi(1-$ $\phi)$ and $\omega_{\text {ion }}^{( \pm)}\left(\varrho_{ \pm}\right)=\varrho_{ \pm}\left(\ln \varrho_{ \pm}-1-\mu_{ \pm}\right)$as the bulk grand potential densities of the solvent and of the \pm -ions (in the low number density limit), respectively. Here $k_{B} T$ is the thermal energy, $\mu_{\phi} k_{B} T$ and $\mu_{ \pm} k_{B} T$ are the chemical potential difference $\left(\mu_{A}-\mu_{B}\right) k_{B} T$ of the solvent particles and the chemical potentials of the \pm -ions, respectively, and $\ell_{B} \widetilde{a}=e^{2} /\left(4 \pi \varepsilon_{0} k_{B} T\right)$ is the Bjerrum length for the vacuum permittivity $\varepsilon_{0}$. The temperature-dependent Flory-Huggins parameter $\chi(T)>0$ describes the effective interaction between solvent particles, where the temperature dependence is usually described by the empirical form $\chi(T)=\chi_{S}+\frac{\chi_{H}}{T}$ with the system specific entropic contribution $\chi_{S}$ and the enthalpic contribution $\chi_{H}[27]$. For $\chi(T) \geq \chi\left(T_{c}\right)$ phase separation occurs in the pure, salt-free solvent within a certain range of $\phi$ whereas for $\chi(T)<\chi\left(T_{c}\right)$ the solvent components $A$ and $B$ are miscible in any proportion. A positive (negative) enthalpic contribution $\chi_{H}$ corresponds to an upper (lower) critical demixing point. The gradient term $\propto(\nabla \phi(z))^{2}$ with $\nabla=\widetilde{a} \widetilde{\nabla}$ penalizes the spatial variation of the solvent composition [28]. The ion-solvent interaction is described within a local density approximation (LDA) by the effective ion potential $k_{B} T V_{ \pm}(\phi)$ generated by the solvent (see below). The relative permittivity $\varepsilon(\phi(\mathbf{r}))$ is assumed to depend locally on the composition of the solvent $\phi(\mathbf{r})$ but not on the ion densities $\varrho_{ \pm}(\mathbf{r})$, which is justified for small ionic strengths, i.e., $\varrho_{ \pm}(\mathbf{r}) \ll 1$. Here the mixing formula $\varepsilon(\phi)=\varepsilon_{A} \phi+\varepsilon_{B}(1-\phi)$ introduced by Böttcher [29] is used [5, 19, 21]. Using SI-units, the electric displacement $\widetilde{\mathbf{D}}=\mathbf{D} e \widetilde{a}^{-2}$ in Eq. (1) fulfills Gauss' law $\nabla \cdot \mathbf{D}\left(\mathbf{r},\left[\varrho_{ \pm}\right]\right)=\varrho_{+}(\mathbf{r})-\varrho_{-}(\mathbf{r}), \mathbf{r} \in \mathcal{V}$, with fixed surface charges $\mathbf{n}(\mathbf{r}) \cdot \mathbf{D}\left(\mathbf{r},\left[\varrho_{ \pm}\right]\right)=\sigma(\mathbf{r}), \mathbf{r} \in \partial \mathcal{V}$, where $\mathbf{n}$ is the unit vector perpendicular to $\partial \mathcal{V}$ pointing towards the exterior of $\mathcal{V}$ (see Ref. [4]). Note that $\mathbf{D}\left(\mathbf{r},\left[\varrho_{ \pm}\right]\right)$is generated by the \pm -ions and the given surface charges $\sigma$; it does not depend explicitly on $\phi$. Within the present model, besides being confined, ions interact with the walls only electrostatically.

Note that by using the square-gradient form of Eq. (1) 

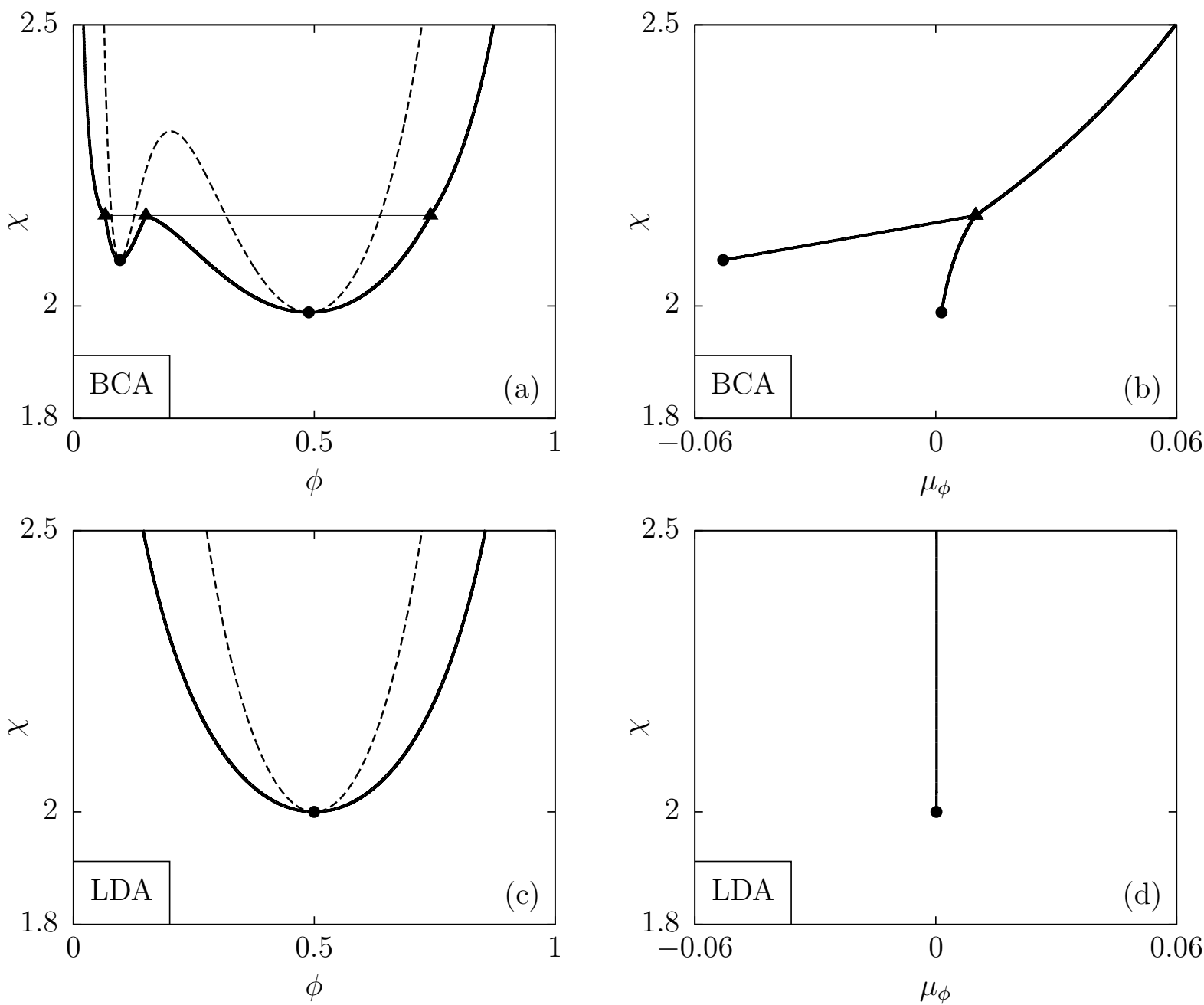

FIG. 1: Bulk phase diagrams of a binary liquid mixture with added salt of constant chemical potential $\left(\right.$ per $\left.k_{B} T\right) \mu_{I}=\mu_{+}+\mu_{-}$ within the bilinear coupling approximation (BCA) $V_{ \pm}^{(B C A)}(\phi)=f_{ \pm} \phi[(\mathrm{a})$ and (b)] and within the local density approximation (LDA, see Eq. (3)) $V_{ \pm}(\phi)=-\ln \left(1-\phi\left(1-\exp \left(-f_{ \pm}\right)\right)\right)[(\mathrm{c})$ and (d)] in terms of the Flory-Huggins parameter $\chi$ and the composition $\phi\left[(\mathrm{a})\right.$ and (c)] or the chemical potential (per $\left.k_{B} T\right) \mu_{\phi}=\mu_{A}-\mu_{B}$ conjugate to the composition $\phi$ of the binary solvent $[(\mathrm{b})$ and $(\mathrm{d})]$. The thick solid lines correspond to the binodals, which delimit the two-phase coexistence regions in the $\phi-\chi$ diagrams [(a) and (c)] from below. The dashed lines are the spinodals and the thin horizontal line in panel (a) is the tie-line corresponding to the triple point $(\boldsymbol{\Delta})$ found within BCA. Representative values for the solubility contrasts per $k_{B} T,\left(f_{+}, f_{-}\right)=(3,26)$, have been chosen. The chemical potential $\mu_{I} k_{B} T$ of the salt corresponds to an ionic strength $\widetilde{I}_{c}=\widetilde{\varrho}_{ \pm}=10 \mathrm{mM}$ at the critical point with composition $\phi=\phi_{c} \approx \frac{1}{2}$. The weak influence of the salt on the phase diagram within LDA leads to curves in panels (c) and (d) which are, on the present scale, almost (but not quite) symmetric with respect to $\phi=\frac{1}{2}$ and $\mu_{\phi}=0$, respectively. Whereas the LDA [(c) and (d)], in agreement with the experimental evidence, exhibits a single critical point $\left(\bullet, \phi_{c, 1} \approx \frac{1}{2}, \chi_{c, 1} \approx 2\right)$, which slightly shifts upon changing the ionic strength (see Fig. 3), the standard BCA $\left[(\mathrm{a})\right.$ and (b)], in contrast to the available experimental observations, leads to a second critical point $\left(\bullet, \phi_{c, 2} \approx 0.1, \chi_{c, 2} \approx 2.1\right)$ as well as to a triple point $(\mathbf{\Lambda})$.

we implicitly assume that the interactions are shortranged [30], i.e., van der Waals forces are not taken into account. Moreover, layering due to packing effects close to walls is also not accounted for by square-gradient theories. Nonetheless such a description provides reliable results at mesoscopic scales [30]. Finally, the ionic strength is assumed to be sufficiently low so that one can neglect short-ranged ion-ion interactions. Therefore the ions interact with each other only via the electrostatic field.
Accordingly, the expression for $\omega_{\text {ion }}^{( \pm)}$does not contain additional Flory-Huggins parameters and there are no square-gradient terms for $\varrho_{ \pm}$. However, these features of the simple functional in Eq. (1) are not expected to lessen the main conclusions of our study, which is devoted to investigate the kind of influences of ions on solvent properties, rather than to construct models with quantitative predictive power. 


\section{B. Ion-solvent interaction}

In Eq. (1) the ion-solvent interaction is described, within a local density approximation (LDA), by a solvent-induced ion potential, $V_{ \pm}(\phi) k_{B} T$. The bilinear coupling approximation (BCA) used in previous investigations (see, e.g., Refs. [5-8, 10-12, 19, 21]) corresponds to the choice $V_{ \pm}^{(\mathrm{BCA})}(\phi):=f_{ \pm} \phi$, where $f_{ \pm} k_{B} T=$ $\left(f_{ \pm A}-f_{ \pm B}\right) k_{B} T$ is the difference between the bulk solvation free energies of a \pm -ion in solvents consisting purely of component $A, f_{ \pm A} k_{B} T$, and purely of component $B, f_{ \pm B} k_{B} T$. The solubility contrasts $f_{ \pm} k_{B} T$ are also known as Gibbs free energies of transfer. In this context the only relevant parameters are the two differences $f_{ \pm}=f_{ \pm A}-f_{ \pm B}$ because the other two independent quantities $f_{ \pm A}+f_{ \pm B}$ can be absorbed as shifts in the definition of the chemical potentials $\mu_{ \pm} k_{B} T$ of the ions. For bulk systems the BCA is identical to the random phase approximation (RPA) [22], which is expected to be reliable only if the coupling strengths are much smaller than the thermal energy, i.e., $\left|f_{ \pm}\right| \ll 1$. However, for electrolyte solutions, this condition is in general not fulfilled. Instead, the Gibbs free energies of transfer between two liquids are usually of the order of some $10 k_{B} T[23,24]$.

Figures 1(a) and 1(b) display the bulk phase diagram for a constant chemical potential (per $k_{B} T$ ) $\mu_{I}:=$ $\mu_{+}+\mu_{-}$of added salt obtained within BCA for the representative values $f_{+}=3, f_{-}=26$. This choice is similar to the Gibbs free energies of transfer for potassium chloride $(\mathrm{KCl})$ from water to acetone: $f_{+}=2, f_{-}=23$ [23]. The condition of local charge neutrality $\varrho_{+}=\varrho_{-}=: I$ in the bulk implies that the ionic strength $I$ depends on the chemical potentials $\mu_{ \pm} k_{B} T$ of the ions only via the sum $\mu_{+}+\mu_{-}=\mu_{I}$. For given uniform composition $\phi$ and ionic chemical potential $\mu_{I}$ the Euler-Lagrange equation of $\Omega$ in Eq. (1) with respect to uniform ion densities $\varrho_{ \pm}=I$ can be used to express the bulk ionic strength as

$$
I_{\mathrm{bulk}}\left(\phi, \mu_{I}\right)=\exp \left(\frac{1}{2}\left(\mu_{I}-V_{+}(\phi)-V_{-}(\phi)\right)\right) .
$$

Within the present model, $I_{\mathrm{bulk}}$ is independent of the Flory-Huggins parameter $\chi$, i.e., it depends on the temperature $T$ only via the normalizations of $\mu_{I}$ and $V_{ \pm}$, which are defined in units of $k_{B} T$. In Fig. 1 the chemical potential $\mu_{I}$ of the salt is fixed such that the solvent composition $\phi=\frac{1}{2}$ leads to an ionic strength $\widetilde{I}=I \widetilde{a}^{-3}=10 \mathrm{mM} \approx 0.006 \mathrm{~nm}^{-3}$, where here and in the following we choose the length scale $\widetilde{a}=2 \AA$. Due to the absence of gradients, electric fields, and surfaces, the bulk phase diagram is determined by the first, third, and fourth term on the right-hand side of Eq. (1). The theoretically predicted occurrence of two critical points $(\bullet)$ as well as of a triple point $(\boldsymbol{\Delta})$ is not supported by experimental evidence, which signals the breakdown of BCA for such large parameters $f_{ \pm}$. Whereas for most systems it is experimentally difficult to preclude the occurrence of such a second critical point or triple point, the experimental resolution is yet sufficiently high to exclude these
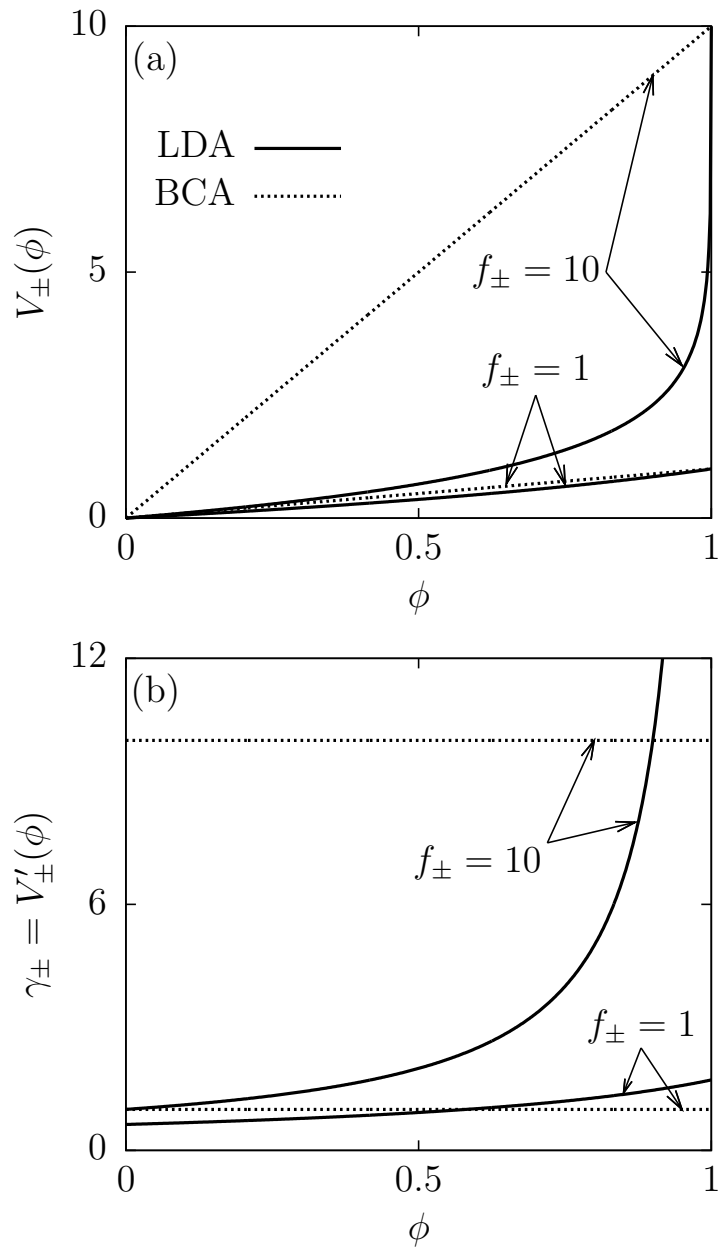

FIG. 2: Comparison of the solvent-induced ion potential $V_{ \pm}(\phi)[(\mathrm{a})]$ and its derivative $\gamma_{ \pm}=V_{ \pm}^{\prime}(\phi)[(\mathrm{b})]$ within LDA and BCA for ion solubility contrast $f_{ \pm}$(see the main text). For small values of $f_{ \pm}$(see the case $f_{ \pm}=1$ ) the differences between LDA and BCA are small. For large values of $f_{ \pm}$(see the case $\left.f_{ \pm}=10\right) V_{ \pm}(\phi)$ and $\gamma_{ \pm}=V_{ \pm}^{\prime}(\phi)$ become large at solvent compositions $\phi \approx 0.5$ within BCA whereas they remain small within LDA. Within LDA $V_{ \pm}^{\prime}(\phi=0)=1-\exp \left(-f_{ \pm}\right)$ and $V_{ \pm}^{\prime}(\phi=1)=\exp \left(f_{ \pm}\right)-1$, while within BCA $V_{ \pm}^{\prime}(\phi)=f_{ \pm}$.

features to occur visibly to the extent as predicted by the BCA (Figs. 1(a) and (b)).

A more appropriate approximation for the solventinduced ion potential $V_{ \pm}(\phi) k_{B} T$, which is derived in Appendix A, is given by (see also Fig. 2(a))

$$
V_{ \pm}(\phi)=-\ln \left(1-\phi\left(1-\exp \left(-f_{ \pm}\right)\right)\right) .
$$

For $\left|f_{ \pm}\right| \ll 1$ this expression reduces to the correct asymptotic expression $V_{ \pm}(\phi) \simeq V_{ \pm}^{(\mathrm{BCA})}(\phi)$. In the limit $f_{ \pm} \rightarrow \infty$, i.e., if ions are insoluble in component $A$, $V_{ \pm}(\phi) \simeq-\ln (1-\phi)$, which corresponds to the free energy of the ions dissolving entirely in component $B$ only, which has the volume fraction $1-\phi$. Similarly, in the limit $f_{ \pm} \rightarrow-\infty$, i.e., if the ions are insoluble in component $B, V_{ \pm}(\phi) \simeq f_{ \pm}-\ln \phi$, which is the free energy of 
the ions dissolving entirely in component $A$ only, which has the volume fraction $\phi$ and for which the solvation free energy is $f_{ \pm}$. For the same set of parameters as in Figs. 1(a) and (b), Figs. 1(c) and (d) display the phase diagram within LDA. In agreement with experimental observations, within LDA only a single critical point $(\bullet)$ occurs (see, e.g., the closed loop-binodals in Ref. [17] with only one lower critical demixing point in the presence of an antagonistic salt, i.e., with $f_{+}$and $f_{-}$having opposite signs). Hence one can conclude that the standard BCA, i.e., $V^{(\mathrm{BCA})}(\phi)=f_{ \pm} \phi$, introduces artifacts for too large ion-solvent couplings, $\left|f_{ \pm}\right| \gg 1$, which are absent within the LDA proposed in Eq. (3). Note that within LDA the salt has such a weak influence on the phase diagram that the curves in Figs. 1(c) and (d) are, on that scale, almost (but not quite) symmetric with respect to $\phi=\frac{1}{2}$ and $\mu_{\phi}=0$, respectively, whereas within BCA the influence of salt on the phase diagram is so strong that the curves in Figs. 1(a) and (b) are highly asymmetric.

If $\phi$ deviates slightly from a certain composition $\phi_{0} \in[0,1]$ one has $V_{ \pm}(\phi) \simeq V_{ \pm}\left(\phi_{0}\right)+\gamma_{ \pm}\left(\phi-\phi_{0}\right)$ with the effective coupling strengths $\gamma_{ \pm}:=V_{ \pm}^{\prime}\left(\phi_{0}\right)=$ $\frac{1-\exp \left(-f_{ \pm}\right)}{1-\phi_{0}\left(1-\exp \left(-f_{ \pm}\right)\right)} \in\left[-\frac{1}{\phi_{0}}, \frac{1}{1-\phi_{0}}\right]$ instead of $f_{ \pm}$as in BCA. For, e.g., $\phi_{0}=1 / 2$ one finds $\gamma_{ \pm}=$ $2 \tanh \left(f_{ \pm} / 2\right) \in[-2,2]$, i.e., the use of BCA, which corresponds to $\gamma_{ \pm} \approx f_{ \pm}$, is justified only for Gibbs free energies of transfer per $k_{B} T, f_{ \pm}$, not larger than 2 (see Fig. 2(b)). However, in previous investigations BCA has been used even for large values of $\left|f_{ \pm}\right|[5-8,19,21]$.

\section{BULK SYSTEMS}

Bulk properties such as the phase diagram and partial structure factors are determined routinely in order to characterize experimentally the behavior of fluids. The available experimental data offer the possibility to assess the quality of the proposed LDA (see the previous Sec. II) with respect to predictions of bulk properties and to compare it with the frequently used BCA.

\section{A. Critical point}

There is experimental evidence [31] that in the phase diagram of a binary liquid mixture the critical point shifts upon adding salt. The direction as well as the magnitude of the shift depend on the materials properties of the binary liquid mixture and of the ions. Due to the relations $\varrho_{A}=\phi, \varrho_{B}=1-\phi, \varrho_{+}=$ $\varrho_{-}=I$ the bulk system, which comprises four particle species, is de facto a binary mixture, characterized by $\mu_{\phi}, \mu_{I}=\mu_{+}+\mu_{-}$, and $\chi$ (i.e., $T$ ). Hence in this three-dimensional space of thermodynamic variables there is a sheet of first-order demixing phase transitions $\left(\mu_{\phi}^{(\text {demix })}\left(\mu_{I}, \chi\right), \mu_{I}, \chi\right)$ bounded by a line of crit-
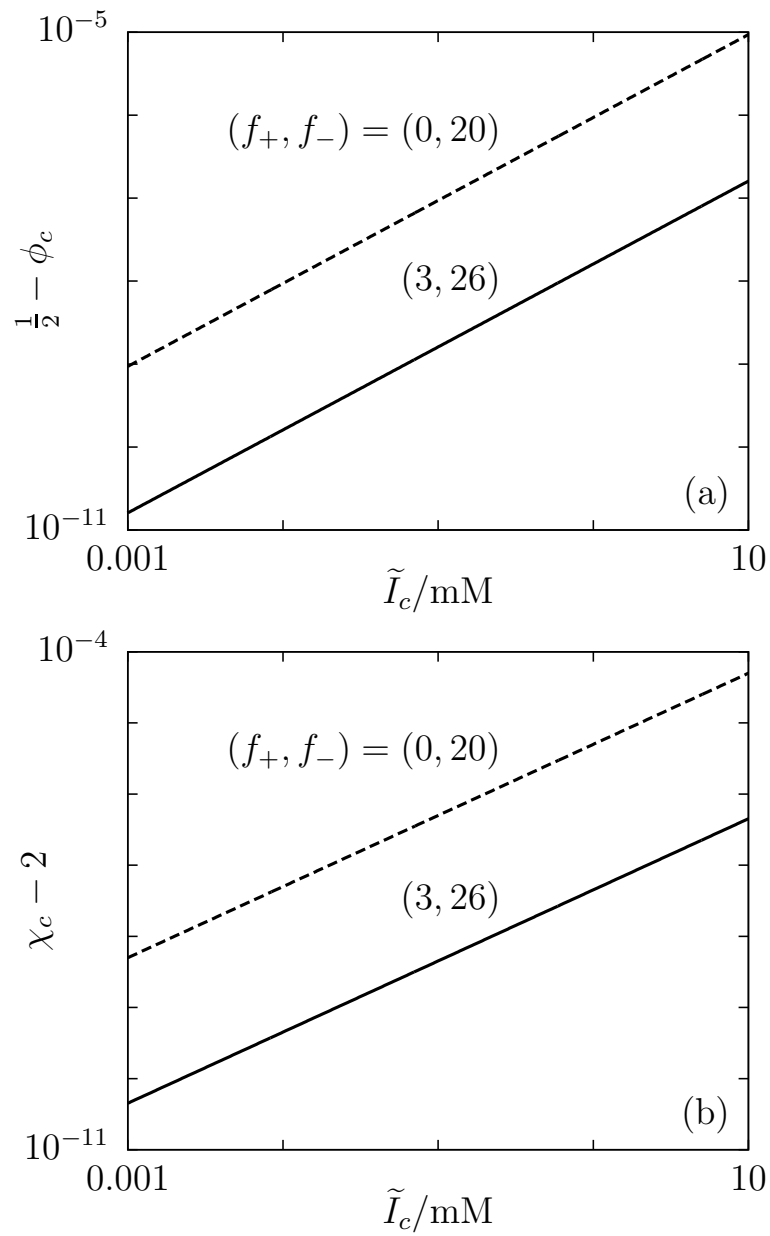

FIG. 3: Variation of the critical volume fraction $\phi_{c}[(\mathrm{a})]$ and the critical Flory-Huggins parameter $\chi_{c}[(b)]$ as function of the ionic strength $\widetilde{I}_{c}=I_{c} \widetilde{a}^{-3}$ at the critical point for two representative sets of solubility contrasts: $\left(f_{+}, f_{-}\right)=(3,26)$ and $(0,20)$. These results show that both $\frac{1}{2}-\phi_{c}$ and $\chi_{c}-2$ depend linearly on $I_{c}$ and that there are no quantitatively significant shifts of the critical point upon varying the ionic strength within experimentally reasonable ranges. On this scale, the phase diagrams for $\left(f_{+}, f_{-}\right)=(3,26)$ (see Figs. $1(\mathrm{c})$ and $\left.(\mathrm{d})\right)$ and for $\left(f_{+}, f_{-}\right)=(0,20)$ are almost indistinguishable. Note that $\phi_{c}\left(\widetilde{I}_{c} \rightarrow 0\right)=\frac{1}{2}$ and $\chi_{c}\left(\widetilde{I}_{c} \rightarrow 0\right)=2$.

ical points $\left(\mu_{\phi}^{(\mathrm{crit})}\left(\mu_{I}\right), \mu_{I}, \chi^{(\mathrm{crit})}\left(\mu_{I}\right)\right)$ which translates into $\left(\phi_{c}, I_{c}, \chi_{c}\right) \equiv\left(\phi^{(\mathrm{crit})}\left(\mu_{I}\right), I^{(\mathrm{crit})}\left(\mu_{I}\right), \chi^{(\mathrm{crit})}\left(\mu_{I}\right)\right)$. For a given chemical potential $\mu_{I} k_{B} T$ the critical point $\left(\phi_{c}, I_{c}, \chi_{c}\right)$ is determined as the minimum of the FloryHuggins parameter $\chi_{s}\left(\phi, I_{\mathrm{bulk}}\left(\phi, \mu_{I}\right)\right)$ at the spinodal as a function of $\phi$ for constant $\mu_{I}$. The spinodal is defined by the set of points $\left(\phi, I, \chi_{s}(\phi, I)\right)$ in the bulk phase diagram for which points $(\phi, I, \chi)$ with $\chi>\chi_{s}(\phi, I)$ exhibit no longer at least a local minimum of the density functional Eq. (1) (see the dashed lines in Figs. 1(a) and (c)). Accordingly, at the spinodal the Hessian matrix of the bulk grand potential density $\Omega(\phi, I) / V$ corresponding to 
Eq. (1) has a zero eigenvalue. This condition leads to

$$
\begin{gathered}
\chi_{s}(\phi, I)=\frac{1}{2}\left(\frac{1}{\phi}+\frac{1}{1-\phi}+\right. \\
\left.\left(V_{+}^{\prime \prime}(\phi)+V_{-}^{\prime \prime}(\phi)-\frac{1}{2}\left(V_{+}^{\prime}(\phi)+V_{-}^{\prime}(\phi)\right)^{2}\right) I\right) .
\end{gathered}
$$

By inverting the relation $I_{c}=I^{(\mathrm{crit})}\left(\mu_{I}\right)$ one obtains $\mu_{I}=$ $\mu_{I}^{\text {(crit) }}\left(I_{c}\right)$. Figure 3 displays the variation of (a) the critical volume fraction $\phi_{c}=\phi^{(\mathrm{crit})}\left(\mu_{I}^{\text {(crit) }}\left(I_{c}\right)\right)$ and (b) the critical Flory-Huggins parameter $\chi_{c}=\chi^{(\text {crit })}\left(\mu_{I}^{(\text {crit })}\left(I_{c}\right)\right)$ as functions of the ionic strength $\widetilde{I}_{c}=I_{c} \widetilde{a}^{-3}$ at the critical point within the present LDA model. Without added salt $(I=0)$ one obtains the critical point $\left(\phi_{c}, \chi_{c}\right)=\left(\frac{1}{2}, 2\right)$ of the pure solvent. For the given choice of the parameters $\left(f_{+}, f_{-}\right)$and for small $I_{c}$ the critical composition $\phi_{c}$ decreases and the critical Flory-Huggins parameter $\chi_{c}$ increases linearly upon increasing the ionic strength $I_{c}$. For small $I_{c}$ the asymptotically linear dependence of the critical point $\left(\phi_{c}, \chi_{c}\right)$ on the ionic strength $I_{c}$ is in agreement with experimental evidence [31, 32]. However, the magnitudes of these shifts are tiny, even for large differences in the solubility contrasts, e.g., $\left(f_{+}, f_{-}\right)=(0,20)$, to the effect that the bulk phase diagrams are almost indistinguishable within experimentally relevant ranges of ionic strengths $\widetilde{I}_{c} \lesssim 10 \mathrm{mM}$. Although for the parameters used in Fig. 3 the shifts of the critical point $\left(\phi_{c}, \chi_{c}\right)$ as predicted within the BCA are up to three orders of magnitude larger than within the LDA, the effect is still small. Within the range of ionic strengths considered in Fig. 3 the experimentally observed critical point shifts are also small [31]. However, significant shifts of the critical temperature $T_{c}$ have been detected for large ionic strengths $\widetilde{I}_{c} \gg 100 \mathrm{mM}$ [31]. But for such large ionic strengths the model in Eq. (1) is not expected to be applicable, because it neglects short-ranged ion-ion interactions.

\section{B. Correlation functions}

The bulk structure of fluids, which is experimentally accessible by X-ray and neutron scattering, provides information complementary to those which follow from the bulk phase behavior. Hence it provides additional opportunities to assess the quality of the LDA. Here we consider a spatially uniform equilibrium state $(\phi, I, \chi)$ in the one-phase region of the phase diagram (see Fig. 1(c)), which minimizes the density functional $\Omega$ in Eq. (1) in the absence of surfaces, i.e., without the last term therein. The corresponding two-point correlation functions $G_{i j}(r)=\varrho_{i} \varrho_{j} h_{i j}(r)=\varrho_{i} \varrho_{j}\left(g_{i j}(r)-\right.$ 1), $i, j \in\{\phi,+,-\}, \varrho_{\phi}:=\phi, \varrho_{ \pm}=I$, are obtained from $G_{i j}(r)=G_{i j}(\mathbf{r}, \mathbf{0}), r=|\mathbf{r}|$, with the inverse $G_{i j}^{-1}\left(\mathbf{r}, \mathbf{r}^{\prime}\right)=$ $\frac{\delta^{2} \Omega}{\delta \varrho_{i}(\mathbf{r}) \delta \varrho_{j}\left(\mathbf{r}^{\prime}\right)}$, where $\sum_{j} \int_{\mathcal{V}} \mathrm{d}^{3} r^{\prime} G_{i j}^{-1}\left(\mathbf{r}, \mathbf{r}^{\prime}\right) G_{j k}\left(\mathbf{r}^{\prime}, \mathbf{r}^{\prime \prime}\right)=$ $\delta_{i k} \delta\left(\mathbf{r}-\mathbf{r}^{\prime \prime}\right)$. The three-dimensional Fourier transforms $\widehat{G}_{i j}(k):=\frac{4 \pi}{k} \int_{0}^{\infty} \mathrm{d} r r G_{i j}(r) \sin (k r)$ with dimensionless $k$, which are proportional to the partial structure factors [22], are given by

$$
\begin{aligned}
\widehat{G}_{\phi \phi}(k) & =\frac{1}{L(k)}\left(k^{2}+\kappa^{2}\right), \\
\widehat{G}_{\phi \pm}(k) & =-\frac{I}{L(k)}\left(V_{ \pm}^{\prime}(\phi) k^{2}+\frac{\kappa^{2}}{2}\left(V_{+}^{\prime}(\phi)+V_{-}^{\prime}(\phi)\right)\right), \\
\widehat{G}_{ \pm \pm}(k) & =\frac{I}{L(k)}\left(\left(\frac{1}{\phi}+\frac{1}{1-\phi}-2 \chi+I\left(V_{+}^{\prime \prime}(\phi)+V_{-}^{\prime \prime}(\phi)\right)+\frac{\chi}{3} k^{2}\right)\left(k^{2}+\frac{\kappa^{2}}{2}\right)-V_{\mp}^{\prime}(\phi)^{2} I k^{2}\right), \\
\widehat{G}_{ \pm \mp}(k) & =\frac{I}{L(k)}\left(\left(\frac{1}{\phi}+\frac{1}{1-\phi}-2 \chi+I\left(V_{+}^{\prime \prime}(\phi)+V_{-}^{\prime \prime}(\phi)\right)+\frac{\chi}{3} k^{2}\right) \frac{\kappa^{2}}{2}+V_{+}^{\prime}(\phi) V_{-}^{\prime}(\phi)^{2} I k^{2}\right)
\end{aligned}
$$

with

$$
\kappa^{2}:=\frac{8 \pi \ell_{B} I}{\varepsilon(\phi)}
$$

as the square of the inverse Debye length and the denominator (see Eq. (5))

$$
\begin{aligned}
L(k):= & \left(k^{2}+\kappa^{2}\right)\left(\frac{\chi}{3} k^{2}+2\left(\chi_{s}(\phi ; I)-\chi\right)\right) \\
& -\frac{I}{2}\left(V_{+}^{\prime}(\phi)-V_{-}^{\prime}(\phi)\right)^{2} k^{2} .
\end{aligned}
$$

Note that $V_{ \pm}^{\prime}(\phi)=0$ leads to $\widehat{G}_{\phi \pm}(k)=0$, i.e., as expected, the fluctuations of the solvent composition and of the ion densities are uncorrelated in the absence of ion-solvent interactions.

Due to the constraint $\varrho_{A}+\varrho_{B}=1$, the correlation functions $\widehat{G}_{A A}(k), \widehat{G}_{A B}(k)$, and $\widehat{G}_{B B}(k)$ of the number density fluctuations of the $A$ and $B$ particles are related to the correlation function $\widehat{G}_{\phi \phi}(k)$ by $\widehat{G}_{A A}(k)=$ $-\widehat{G}_{A B}(k)=\widehat{G}_{B B}(k)=\widehat{G}_{\phi \phi}(k)$. In the following we re- 
fer to $\widehat{G}_{\phi \phi}(k)$ as the solvent structure factor. It can be written in the form

$$
\widehat{G}_{\phi \phi}(k)=\frac{\widehat{G}_{\phi \phi}(0)}{1+(\lambda k)^{2}\left(1-\frac{g^{2}}{1+(k / \kappa)^{2}}\right)}
$$

with

$$
\lambda:=\sqrt{\frac{\chi}{6\left(\chi_{s}(\phi, I)-\chi\right)}}
$$

and

$$
g^{2}:=\frac{3(\Delta \gamma)^{2} \varepsilon(\phi)}{16 \pi \ell_{B} \chi}
$$

where $\Delta \gamma:=\gamma_{+}-\gamma_{-}=V_{+}^{\prime}(\phi)-V_{-}^{\prime}(\phi)$. The isothermal compressibility, which is proportional to $\widehat{G}_{\phi \phi}(0)=$ $\left(2\left(\chi_{s}(\phi, I)-\chi\right)\right)^{-1}$, diverges $\propto\left|\chi-\chi_{c}\right|^{-\gamma}$ upon approaching the critical point $\left(\phi_{c}, I_{c}, \chi_{c}\right)$. As expected within the present mean-field theory, one finds the classical critical exponent $\gamma=1$ instead of $\gamma \approx 1.24$ for the Ising universality class [33]. For a state point $(\phi, I, \chi)$ in the bulk phase diagram (see Figs. 1(a) and (c)) the length $\lambda$ is an (inverse) measure of the deviation of $\chi$ from its value $\chi_{s}(\phi, I)$ at the spinodal. Equation (8) has already been derived in Ref. [5] within BCA, which corresponds to the linear approximation $\Delta \gamma \approx f_{+}-f_{-}$. For $|g| \leq 1$ in Eq. (8) the solvent structure factor $\widehat{G}_{\phi \phi}(k)$ is a monotonically decreasing function of the wave number $k$, whereas for $|g|>1$ at $k_{\max }=\kappa \sqrt{|g|-1}$ a maximum $\widehat{G}_{\phi \phi}\left(k_{\max }\right)=$ $\frac{\widehat{G}_{\phi \phi}(0)}{1-(\kappa \lambda)^{2}(|g|-1)^{2}}$ occurs. Hence, if $|g|>1, \widehat{G}_{\phi \phi}\left(k_{\max }\right)$ diverges as function of $\chi$ at $\lambda=\lambda_{\text {unstable }}=(\kappa(|g|-1))^{-1}$, i.e., the spatially uniform bulk state becomes unstable upon approaching the critical point. Note that in the limits $|g| \rightarrow 0$ (no ion-solvent coupling) or $\kappa \rightarrow 0$ (no salt) Eq. (8) leads to the Ornstein-Zernike-like solvent structure factor $\widehat{G}_{\phi \phi}(k)=\widehat{G}_{\phi \phi}(0) /\left(1+(\lambda k)^{2}\right)$. In this case $\lambda$ can be identified with the bulk correlation length.

Experimental reports of uniform bulk states close to the critical point of water $+2,6$-dimethylpyridine mixtures with $\mathrm{KBr}, \mathrm{KCl}$, and $\mathrm{Mg}\left(\mathrm{NO}_{3}\right)_{2}$ (see Ref. [34]) as well as distributions of neutron scattering intensities of water+3-methylpyridine with $\mathrm{LiCl}, \mathrm{NaCl}, \mathrm{KCl}, \mathrm{NaBr}$, and $\mathrm{MgSO}_{4}$, which vary monotonically as function of $k$ (see Refs. [13, 14]), indicate that in these systems one has $|g|<1$. Within the present LDA this latter relation is expected to be fulfilled: Close to the critical point $\left(\phi_{c}, \chi_{c}\right) \approx\left(\frac{1}{2}, 2\right)$ (see Fig. 3$)$ of, e.g., the widely studied binary liquid mixture of 3-methylpyridine (component $A$, $\varepsilon_{A}=10$ ) and water (component $B, \varepsilon_{B}=80$ ) with a lower critical demixing point at $T_{c} \approx 316 \mathrm{~K}$, i.e., $\ell_{B} \widetilde{a} \approx 529 \AA$, one obtains $|g|<0.3$ independent of the type of salt, because $|\Delta \gamma| \lesssim 4$ (see the last paragraph in Subsec. II B). However, within BCA, i.e., for $\Delta \gamma \approx f_{+}-f_{-}$with typically $\left|f_{+}-f_{-}\right| \gg 1[23,24]$, one has to expect $|g| \gg 1$, which, according to the above reasoning, is in sharp contrast to the available experimental results. We mention that experimental reports [15-17] of "periodic structures" in heavy water +3 -methylpyridine mixtures with sodium tetraphenylborate $\left(\mathrm{NaBPh}_{4}\right)$ cannot, however, be expected to find a consistent interpretation in terms of a local ion solvation model as given in Eq. (1), neither within BCA nor within the present LDA, because the anions $\left(\left[\mathrm{BPh}_{4}\right]^{-}\right)$are much larger than the solvent particles, such that in these systems the ion size is expected to be relevant.

The charge-charge structure factor $S_{Z Z}(k)=$ $\left(\widehat{G}_{ \pm \pm}(k)-\widehat{G}_{ \pm \mp}(k)\right) / I[22]$, which measures correlations of fluctuations $Z$ of the local charge density around $\varrho_{+}-\varrho_{-}=0$, is obtained by inserting the expressions for $\widehat{G}_{ \pm \pm}(k)$ and $\widehat{G}_{ \pm \mp}(k)$ from Eq. (5):

$$
S_{Z Z}(k)=k^{2} \frac{\frac{\chi}{3} k^{2}+2\left(\chi_{s}(\phi, I)-\chi\right)}{L(k)} .
$$

The asymptotic behavior $S_{Z Z}(k \rightarrow 0) \simeq(k / \kappa)^{2}$ is the signature for perfect screening [22]. Further, the case $\Delta \gamma=0$ corresponds to the Debye-Hückel limit $S_{Z Z}(k)=$ $k^{2} /\left(k^{2}+\kappa^{2}\right)$.

The asymptotic behavior of the correlation function $G_{i j}(r)=\frac{1}{2 \pi^{2} r} \int_{0}^{\infty} \mathrm{d} k k \widehat{G}_{i j}(k) \sin (k r)$ can be inferred from a pole analysis of $\widehat{G}_{i j}(k)$, which amounts to determine the roots of the denominator $L(k)$ defined in Eq. (7) $[35,36]$. Since $L(k)$ is a polynomial in $k$ of degree four it has four and only four complex roots $k_{\nu}=k_{\nu}^{\prime}+i k_{\nu}^{\prime \prime}, k_{\nu}^{\prime}=$ $\operatorname{Re}\left(k_{\nu}\right), k_{\nu}^{\prime \prime}=\operatorname{Im}\left(k_{\nu}\right), \nu \in\{1, \ldots, 4\}$. Due to the actual structure of $L(k)$ there are constraints on the locations of the four roots $k_{\nu}$ in the complex plane. If $L\left(k=k_{\nu}\right)$ vanishes this holds also for $k=k_{\nu}^{*}$, because $L(k)$ has real coefficients. Moreover, if $L\left(k=k_{\nu}\right)$ vanishes this also holds for $k=-k_{\nu}$, because $L(k)$ is a polynomial in $k^{2}$. Accordingly this is also true for $k=-k_{\nu}^{*}$. This implies the root structure shown in Fig. 4. Three distinct situations can occur. For purely imaginary roots given by $\left\{k_{1}=i k_{1}^{\prime \prime}, k_{2}=i k_{2}^{\prime \prime}, k_{3}=-k_{1}, k_{4}=-k_{2}\right\}$ with $0<k_{1}^{\prime \prime}<k_{2}^{\prime \prime}$ (see Fig. 4(a)) the asymptotic decay of the two-point correlation functions $G_{i j}(r \rightarrow \infty)$ is monotonic $\propto \exp \left(-k_{1}^{\prime \prime} r\right) / r$. For complex roots $\left\{k_{1}=k_{1}^{\prime}+i k_{1}^{\prime \prime}, k_{2}=\right.$ $\left.-k_{1}^{*}, k_{3}=-k_{1}, k_{4}=k_{1}^{*}\right\}$ with $k_{1}^{\prime}, k_{1}^{\prime \prime}>0$ (see Fig. 4(b)) the two-point correlation functions $G_{i j}(r)$ vary asymptotically $\propto \sin \left(k_{1}^{\prime} r+\right.$ const $) \exp \left(-k_{1}^{\prime \prime} r\right) / r$ giving rise to a damped oscillatory decay. Finally, purely real roots $\left\{k_{1}=k_{1}^{\prime}, k_{2}=k_{2}^{\prime}, k_{3}=-k_{1}, k_{4}=-k_{2}\right\}$ with $0<k_{1}^{\prime}<k_{2}^{\prime}$ (see Fig. 4(c)) indicate an unstable bulk state, i.e., the corresponding point in the phase diagram is located in between the spinodals. The exponential decay of the two-point correlation functions (whether monotonically or oscillatory) is consistent with the short range of the interactions implied by taking a gradient expansion in Eq. (1). 

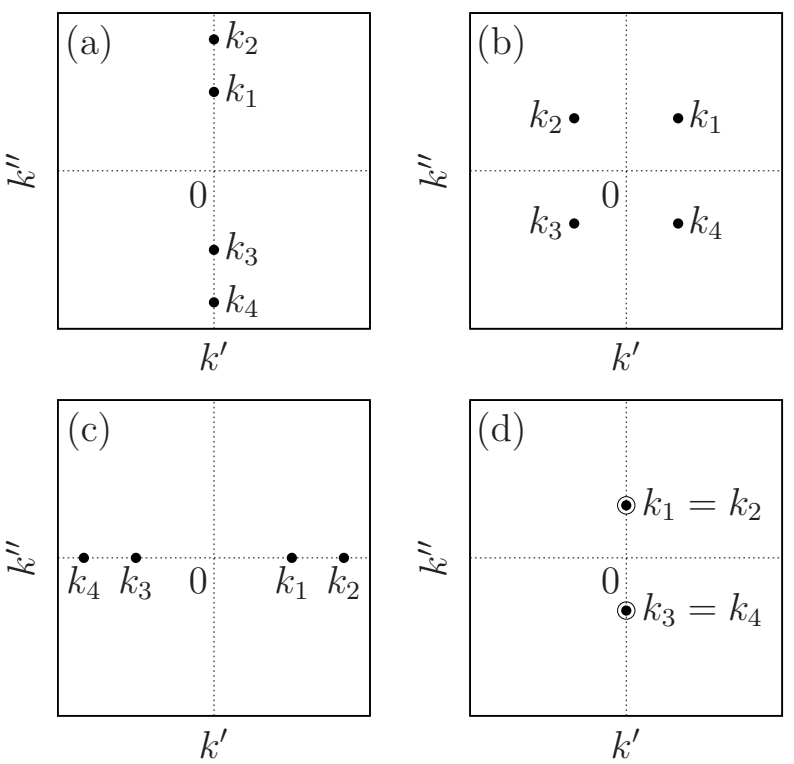

FIG. 4: Poles $k_{1}, \ldots, k_{4}$ of the Fourier transform $\widehat{G}_{i j}(k)$ of the two-point correlation functions $G_{i j}(r)$ in the complex plane $k=k^{\prime}+i k^{\prime \prime} \in \mathbb{C}$, which correspond to the roots of the denominator $L(k)$ (see Eq. (7)). According to the analytic structure of $L(k)$ (see the main text) only the three distinct situations shown in panels (a)-(c) can occur. Purely imaginary poles [(a) correspond to a monotonic decay of $G_{i j}(r \rightarrow \infty)$ whereas a pole structure as in panel (b) ( $\left|k_{\nu}\right|$ all equal) corresponds to an oscillatory decay of $G_{i j}(r \rightarrow \infty)$. Purely real poles $[(c)]$ indicate an unstable bulk state, which does not occur in the one-phase region of the phase diagram in Fig. 1(c). The merging of two poles on the imaginary axis [(d)] corresponds to a Kirkwood crossover point.

Thermodynamic states in the bulk phase diagram with monotonically decaying $G_{i j}(r \rightarrow \infty)$ are separated from states with damped oscillatory decay of $G_{i j}(r \rightarrow \infty)$ by so-called Kirkwood crossover lines [37]. Crossing these lines is associated with the merging of two purely imaginary poles (see Figs. $4(\mathrm{a})$ and (d)) of $\widehat{G}_{i j}(k)$ in the upper (and similarly in the lower) half of the complex plane and with a subsequent emergence of a pair of two poles (see Fig. 4(b)) with equal imaginary parts and with real parts of equal absolute value but of opposite sign [38]. In the phase diagrams of Fig. 5 the Kirkwood crossover lines are denoted by dotted lines and damped oscillatory decay of $G_{i j}(r \rightarrow \infty)$ occurs at state points in the grey area enclosed by the Kirkwood crossover lines. The parameters correspond to the aforementioned binary liquid mixture water+3-methylpyridine $\left(\varepsilon_{A}=10, \varepsilon_{B}=80\right.$, $\ell_{B} \widetilde{a}=529 \AA$ ); for simplicity the temperature dependence of the Bjerrum length $\ell_{B}$ is ignored. The chemical potential $\mu_{I}$ of the salt is fixed such that there is an ionic strength $\widetilde{I}_{c}=10 \mathrm{mM}$ at the (shifted) critical point. Figures $5(\mathrm{a})$ and (c) correspond to the parameters $\left(f_{+}, f_{-}\right)=(3,26)$ used in Figs. 1(a) (BCA) and (c) (LDA), respectively. Figure 5(b) refers to the case of a strongly antagonistic salt, $\left(f_{+}, f_{-}\right)=(-20,20)$, whereas
Fig. $5(\mathrm{~d})$ relates to the intermediate case $\left(f_{+}, f_{-}\right)=$ $(0,20)$. Within BCA (see Fig. 5(a)), the damped oscillatory decay of $G_{i j}(r \rightarrow \infty)$ prevails in a large portion of the phase diagram, and wave lengths of the oscillations as small as the particle size $\widetilde{a}$ can occur at state points in the center of the grey area. However, within the present LDA, damped oscillatory decay of $G_{i j}(r \rightarrow \infty)$ is found only in a narrow range of $\mathcal{O}(\Delta \gamma I)$ for values of $\chi$ around $\chi=\frac{\chi_{s}(\phi, I)}{1+\kappa^{2} / 6}+\mathcal{O}\left((\Delta \gamma)^{2} I\right)$, which extends into the onephase region only in the vicinity of the critical point (see Figs. 5(b)-(d)).

For small wave numbers $k$ the structure factor $\widehat{G}_{\phi \phi}(k \ll \kappa) \simeq \frac{\widehat{G}_{\phi \phi}(0)}{1+(\lambda k)^{2}\left(1-g^{2}\right)}$ (see Eq. (8)) takes the Ornstein-Zernike form $\frac{\widehat{G}_{\phi \phi}(0)}{1+\left(\xi^{(\mathrm{OZ})} k\right)^{2}}$ with the length

$$
\xi^{(\mathrm{OZ})}=\lambda \sqrt{1-g^{2}}=\sqrt{\frac{\chi\left(1-g^{2}\right)}{6\left(\chi_{s}(\phi, I)-\chi\right)}}
$$

which we shall refer to as the Ornstein-Zernike length. This length $\xi^{(\mathrm{OZ})}$ is determined routinely in scattering experiments by fitting an Ornstein-Zernike expression to scattered intensities at small momentum transfer [34]. For water+2,6-dimethylpyridine mixtures with $\mathrm{KBr}, \mathrm{KCl}$, and $\mathrm{Mg}\left(\mathrm{NO}_{3}\right)_{2}$ (see Ref. [34]) it has been found experimentally that the amplitude $\xi_{0}^{(\mathrm{OZ})}$ of $\xi^{(\mathrm{OZ})}=$ $\xi_{0}^{(\mathrm{OZ})}\left|\left(T-T_{c}\right) / T_{c}\right|^{-\nu}$ is to a large extent independent of the considered type of salt and ionic strength. Due to $\xi_{0}^{(\mathrm{OZ})} \propto \sqrt{1-g^{2}}$ this observation indicates that one has $g^{2} \ll 1$, which, according to the arguments given above, is expected within LDA but is not compatible with predictions following from BCA.

The poles $\left\{k_{1}, \ldots, k_{4}\right\}$ of the solvent structure factor $\widehat{G}_{\phi \phi}(k)$ can be expressed in terms of the Ornstein-Zernike length $\xi^{(\mathrm{OZ})}$ and the inverse Debye length $\kappa$. For a monotonic decay of $G_{\phi \phi}(r \rightarrow \infty)$ one has purely imaginary poles $k_{\nu}=i k_{\nu}^{\prime \prime}$ with

$$
k_{1}^{\prime \prime}=-k_{3}^{\prime \prime}=\sqrt{2\left(u-\sqrt{u^{2}-v^{2}}\right)}
$$

and

$$
k_{2}^{\prime \prime}=-k_{4}^{\prime \prime}=\sqrt{2\left(u+\sqrt{u^{2}-v^{2}}\right)},
$$

whereas a damped oscillatory decay of $G_{\phi \phi}(r \rightarrow \infty)$ is characterized by the poles at $\left\{k_{1}=k_{1}^{\prime}+i k_{1}^{\prime \prime}, k_{2}=\right.$ $\left.-k_{1}^{*}, k_{3}=-k_{1}, k_{4}=k_{1}^{*}\right\}$ with

$$
k_{1}^{\prime}=\sqrt{v-u}, \quad k_{1}^{\prime \prime}=\sqrt{v+u},
$$

where

$$
u:=\frac{\kappa^{2}\left(1-g^{2}\right)}{4}\left(1+\frac{1}{\left(\kappa \xi^{(\mathrm{OZ})}\right)^{2}}\right)
$$



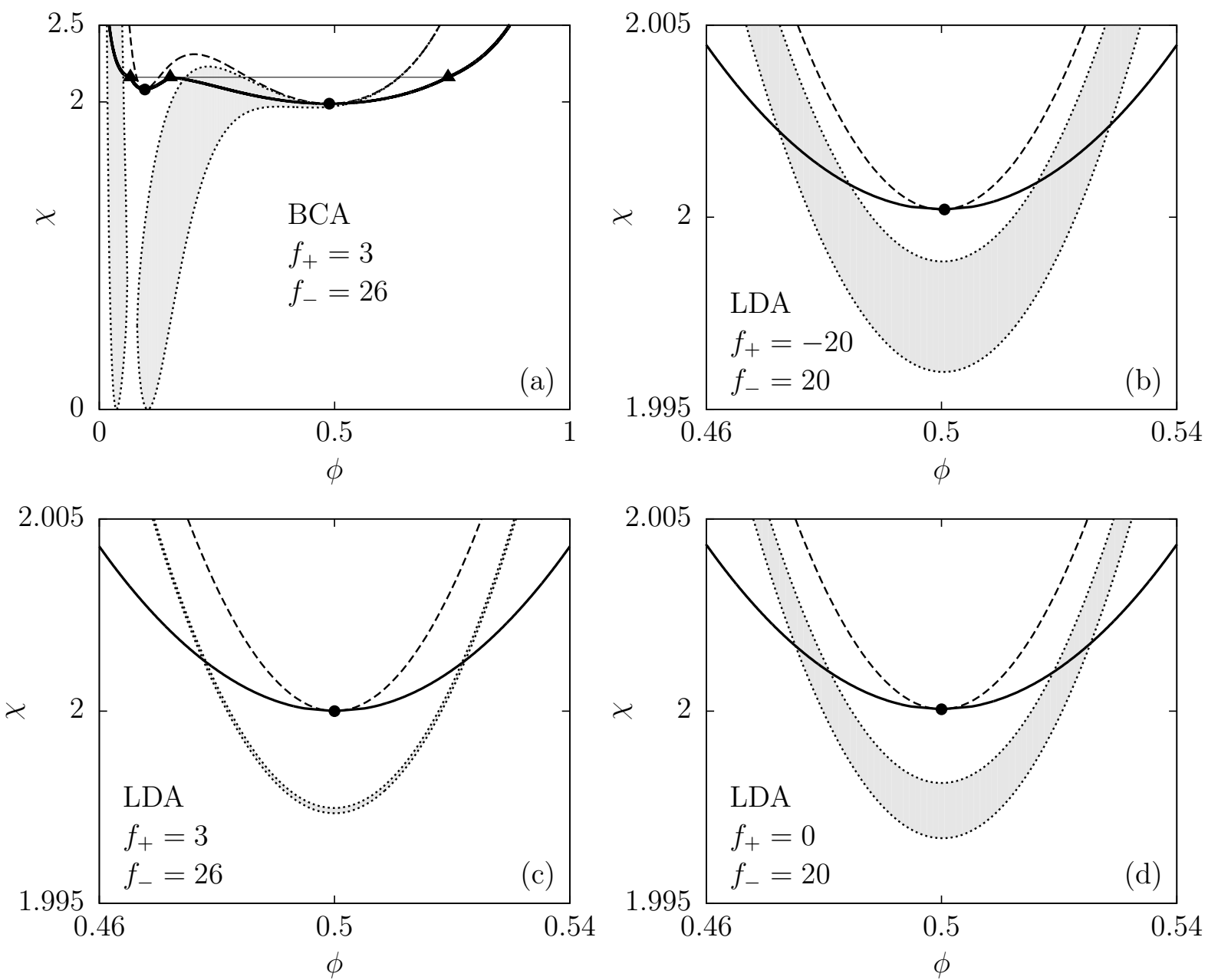

FIG. 5: Phase diagrams as in Fig. 1 with Kirkwood crossover lines (dotted lines) within the bilinear coupling approximation (BCA) $[(\mathrm{a})]$ and the local density approximation (LDA) $[(\mathrm{b})-(\mathrm{d})]$. The parameters correspond to the binary liquid mixture water+3-methylpyridine $\left(\varepsilon_{A}=10, \varepsilon_{B}=80, \ell_{B} \widetilde{a}=529 \AA\right)$; for simplicity the temperature dependence of the Bjerrum length $\ell_{B}$ is ignored. The chemical potential $\mu_{I} k_{B} T$ of the salt is fixed such that at the (slightly shifted) critical point with composition $\phi=\phi_{c} \approx \frac{1}{2}$ there is an ionic strength $\widetilde{I}_{c}=10 \mathrm{mM}$. Outside the grey regions bounded by the dotted lines the two-point correlation functions exhibit asymptotically a monotonic decay, whereas inside these regions damped oscillatory decays occur. Within BCA a large portion of the phase diagram corresponds to oscillatory decay, whereas within LDA this occurs only in a narrow band within which the value of the bulk correlation length is close to that of the Debye screening length. Note the differences in scales for the axes in (a) and in (b)-(d).

and

$$
v:=\frac{\kappa \sqrt{1-g^{2}}}{2 \xi^{(\mathrm{OZ})}}
$$

Close to the critical point (i.e., for $\xi^{(\mathrm{OZ})} \rightarrow \infty$ ) one finds a monotonic decay of $G_{\phi \phi}(r \rightarrow \infty)$ with the decay length $1 / k_{1}^{\prime \prime} \simeq \xi^{(\mathrm{OZ})} \propto\left|\chi-\chi_{c}\right|^{-\nu}$ (see Eq. (13)) with the meanfield critical exponent $\nu=\frac{1}{2}$ instead of $\nu \approx 0.63$ for the Ising universality class [33]. Therefore the electrostatic interactions do not affect the universal critical exponent, but they can influence the non-universal critical amplitude $\xi_{0}^{(\mathrm{OZ})}$.

Figure 6 displays the real and imaginary parts of the poles $k_{\nu}$ of $\widehat{G}_{i j}(k)$ in the ranges $k_{\nu}^{\prime}=\operatorname{Re}\left(k_{\nu}\right), k_{\nu}^{\prime \prime}=$
$\operatorname{Im}\left(k_{\nu}\right) \geq 0$ at the critical composition $\phi=\phi_{c} \approx \frac{1}{2}$ for the parameters corresponding to Fig. 5(b). The four poles $\left\{k_{1}, \ldots, k_{4}\right\}$ can be expressed in terms of $\left(k_{1}^{\prime}, k_{1}^{\prime \prime}, k_{2}^{\prime \prime}\right)$ (see Eqs. (13)-(15)). In Fig. 6 the two purely imaginary poles $k_{1}$ and $k_{2}$ with positive imaginary parts for monotonically decaying $G_{i j}(r \rightarrow \infty)$ occur as two branches, which merge at the Kirkwood crossover points $(\bullet)$. From Eqs. (13) and (14) the Kirkwood crossover points (•) are characterized by $u=v$ which leads to (see Eqs. (16) and (17)) $\kappa \xi^{(\mathrm{OZ})}=\sqrt{\frac{2}{1 \pm g}-1} \approx 1$. Hence at the Kirkwood crossover points the inverse decay lengths of $G_{i j}(r \rightarrow \infty)$ correspond approximately to $\kappa$ (dashed line) and $1 / \xi^{(\mathrm{OZ})}$ (dotted line). At the critical point $\left(\chi=\chi_{c}\right.$, i.e., $k_{1}^{\prime \prime}=0$, see Eqs. (13) and (17)), $G_{i j}(r \rightarrow \infty)$ decays as $1 / r$ with 


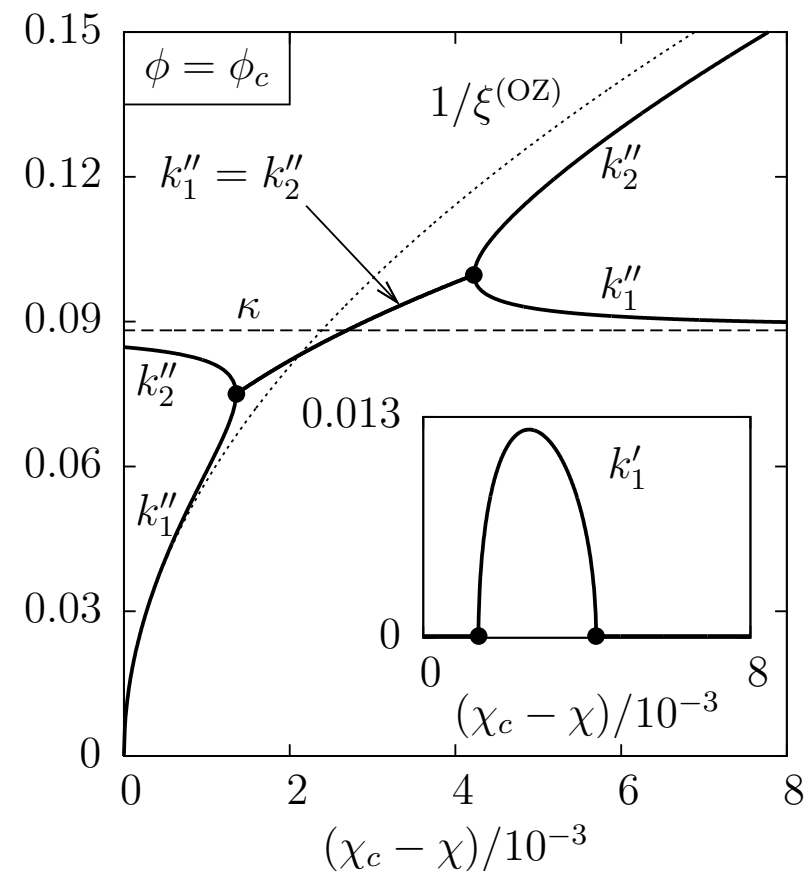

FIG. 6: Within LDA real and imaginary parts of the poles $k_{\nu}=k_{\nu}^{\prime}+i k_{\nu}^{\prime \prime}, k_{\nu}^{\prime}=\operatorname{Re}\left(k_{\nu}\right), k_{\nu}^{\prime \prime}=\operatorname{Im}\left(k_{\nu}\right), \nu \in\{1, \ldots, 4\}$, of the Fourier transform $\widehat{G}_{i j}(k)$ of the two-point correlation functions $G_{i j}(r)$ as functions of the deviation $\chi_{c}-\chi$ from the critical point at the critical composition $\phi=\phi_{c} \approx \frac{1}{2}$ for the parameters corresponding to Fig. 5(b). The four poles $k_{1}, \ldots, k_{4}$ can be expressed in terms of $\left(k_{1}^{\prime}, k_{1}^{\prime \prime}, k_{2}^{\prime \prime}\right)$ (see Eqs. (13)-(17) and Fig. 4). If $G_{i j}(r \rightarrow \infty)$ decays monotonically, the poles of $\widehat{G}_{i j}(k)$ are purely imaginary $\left(k_{1}^{\prime}=0\right)$, giving rise to two branches $k_{1}^{\prime \prime}$ and $k_{2}^{\prime \prime}$ of positive imaginary parts (see Fig. 4(a)). If $G_{i j}(r \rightarrow \infty)$ decays oscillatorily, there is only one pole of $\widehat{G}_{i j}(k)$ with positive real and imaginary parts $\left(k_{2}^{\prime \prime}=k_{1}^{\prime \prime}\right)$ (see Fig. $\left.4(\mathrm{~b})\right)$. The merging of the two branches $k_{1}^{\prime \prime}$ and $k_{2}^{\prime \prime}$ for monotonic asymptotic decay takes place at the Kirkwood crossover points $(\bullet)$ (see Fig. 4(d)). Upon varying $\phi$ these points form the Kirkwood crossover lines (dotted lines in Fig. 5). For comparison the inverse Debye length $\kappa$ (dashed line) as well as the inverse OrnsteinZernike length $1 / \xi^{(\mathrm{OZ})} \propto \sqrt{\chi_{c}-\chi}($ dotted line, see Eq. (12)) are displayed. Within the range of values of $\chi$ leading to an oscillatory decay, depicted by the grey regions in Fig. 5, one has $\kappa \approx 1 / \xi^{(\mathrm{OZ})}$. Within the range of monotonic decay the decay rate of the leading contribution to $G_{i j}(r \rightarrow \infty)$ is given by $k_{1}^{\prime \prime}$ whereas that of the subdominant contribution is $k_{2}^{\prime \prime}$. For $\chi_{c}-\chi \leq 1.3 \times 10^{-3}$ the decay rates are $k_{1}^{\prime \prime} \approx 1 / \xi^{(\mathrm{OZ})}$ and $k_{2}^{\prime \prime} \approx \kappa$, whereas for $\chi_{c}-\chi \geq 4.3 \times 10^{-3}$ the decay rates are $k_{1}^{\prime \prime} \approx \kappa$ and $k_{2}^{\prime \prime} \approx 1 / \xi^{(\mathrm{OZ})}$.

a subdominant contribution $\propto \exp \left(-\kappa r \sqrt{1-g^{2}}\right) / r$ (see Fig. 6), i.e., as anticipated above, the leading decay at large distances is governed by the vicinity to the critical point, whereas the ion-solvent coupling manifests itself in the corrections to the leading behavior. Further away from the critical point the leading contribution decays $\propto \exp \left(-k_{1}^{\prime \prime} r\right) / r$ with a subdominant contribution $\propto \exp \left(-k_{2}^{\prime \prime} r\right) / r$ (see Fig. 6). The inset of Fig. 6 dis- plays the absolute value of the real parts $\left|k_{\nu}^{\prime}\right|=k_{1}^{\prime}$ of the poles of $\widehat{G}_{i j}(k)$, which is identical to the wave number $k_{1}^{\prime}$ (see Eq. (15)) of the oscillatory part of $G_{i j}(r)$ and which is non-zero within the grey region of Fig. 5(b). For the strongly antagonistic salt with $f_{+}=-20$ and $f_{-}=20$, in Fig. 5(b) the shortest wave length of the oscillations is given by $\left(2 \pi / k_{1}^{\prime}\right)_{\min } \approx 513$ (see the inset in Fig. 6). The corresponding value of $k_{1}^{\prime \prime}$ is $\approx 0.0873$ so that $\left(2 \pi / k_{1}^{\prime}\right)_{\min } \approx 45 / k_{1}^{\prime \prime}$, i.e., $G_{i j}(r) \propto \sin \left(k_{1}^{\prime} r+\right.$ const) $\exp \left(-k_{1}^{\prime \prime} r\right) / r$ decays already within $1 / 45$ of a period. In less extreme cases of solubility contrasts $f_{ \pm}$, such as those in Figs. 5(c) and (d), the shortest wave lengths are even larger. Therefore, within LDA, the oscillations in $G_{i j}(r)$, if they occur, are not expected to be experimentally detectable. In contrast, as already mentioned above, within BCA it is possible that the shortest wave lengths of the oscillations are of the order of the particle size; such an asymptotic oscillatory decay can be expected to be visible in the pair distribution function. However, we are not aware of any experimental reports of Kirkwood crossover lines, which is in line with the results obtained within the LDA.

\section{SEMI-INFINITE SYSTEMS BOUNDED BY A PLANAR WALL}

Interfacial properties such as number density profiles and the excess adsorption are experimentally accessible, albeit requiring more effort than determining bulk properties. However, if available, they provide information which is significantly more detailed than the one which can be inferred from the bulk properties discussed in the previous Sec. III. In this respect the simplest and experimentally appealing setting is that of a semi-infinite planar system, on which we shall focus in the following. Predictions within the proposed LDA (see Sec. II) will be compared with those obtained within the BCA as well as with the limited amount of corresponding experimental data which are presently available. Additional experimental settings are proposed in order to assess the predictive power of the LDA.

\section{A. Profiles}

According to the fluctuation-dissipation theorem the linear density response of a system to a weak external field is determined by the two-point correlation functions of the unperturbed system [22]. Therefore, the number density profiles far from a wall exhibit asymptotically the same type of decay towards their bulk values, i.e., either monotonically or damped oscillatorily, with the same decay length and periodicity as the two-point bulk correlation functions $G_{i j}(r)$. (Note that this correspondence does no longer hold in the presence of algebraically decaying interaction potentials [39], which we do not consider here.) However, from this argument one cannot draw 
reliable conclusions concerning their structure close to the wall. Therefore in this latter range we determine the structure numerically for particular sets of parameters. Moreover, close to the wall packing effects due to the finite size of the fluid particles lead to layering which extends a few particle diameters into the system. However, this kind of structure is not captured by the present square-gradient model (Eq. (1)).

The solid lines in Fig. 7 correspond to the composition $\phi(z)[(\mathrm{a})]$, the electrostatic potential $\widetilde{\psi}(z)=\psi(z) k_{B} T / e$ with $\varepsilon(\phi) \psi^{\prime}(z)=-4 \pi \ell_{B} D(z)$ and $\psi(z \rightarrow \infty) \rightarrow 0$ $[(\mathrm{b})]$, the cation number density $\varrho_{+}(z)[(\mathrm{c})]$, and the anion number density $\varrho_{-}(z)[(d)]$ in a semi-infinite system bounded by a wall positioned at $z=0$ with surface charge density $\sigma$ and surface field strength $h$, as obtained from numerically minimizing the density functional in Eq. (1). The solvent permittivity is chosen to resemble that of a mixture of 3-methylpyridine (component $A, \varepsilon_{A}=10$ ) and water (component $B, \varepsilon_{B}=80$ ). The composition profiles $\phi(z)$ in Fig. 7(a) turn out to be monotonic for weak ( $h= \pm 0.01$ ) as well as for strong surface fields $(h= \pm 1)$. Due to the negative surface charge density $\sigma$, the monotonic electrostatic potential profile $\psi(z)$ in Fig. $7(\mathrm{~b})$ is negative with surface potentials $\widetilde{\psi}(0)$ of some tens of $\mathrm{mV}$, which is a common order of magnitude [40]. Within the range $0 \leq z \leq 2$ close to the wall the electrostatic potential $\psi$ becomes less negative upon changing the surface field strength from $h=1$ to $h=-1$ due to the increase of the permittivity as a result of the increase of the volume fraction $1-\phi$ of component $B$ close to the wall. Similarly, due to the negative surface charge, close to the wall the number density $\varrho_{+}$of the cations in Fig. 7(c) is larger and the number density $\varrho_{-}$ of the anions in Fig. $7(\mathrm{~d})$ is smaller than in the bulk. Upon changing the surface field strength from $h=1$ to $h=-1$, close to the wall the number density $\varrho_{+}$of the cations decreases and that of the anions, $\varrho_{-}$, increases. This feature follows partly from the variation of the electrostatic potential $\psi$. In addition, for the current choice of parameters the anions dissolve better in component $B$ than in component $A$ of the solvent $\left(f_{-}>0\right)$, such that the component $B$ enriched near the surface (see Fig. 7(a)) mediates a certain preference of the anions for the wall.

The dashed lines in Fig. 7(a) correspond to the approximate profile

$$
\begin{aligned}
\bar{\phi}(z) & =\phi_{b}+\frac{C_{G L}}{\sinh \left(\left(z+z_{0}\right) / \xi\right)}, \\
C_{G L} & :=\operatorname{sign}(h) \sqrt{\frac{\chi}{8 \xi^{2}}}
\end{aligned}
$$

with the extrapolation length $z_{0}$. Here and in the following we call $\xi \equiv \xi^{(\mathrm{OZ})}$ (see Sec. III B) the bulk correlation length. As noted in Sec. III B, close to the critical point $1 / \xi$ corresponds to the asymptotic decay rate $k_{1}^{\prime \prime}$ of the solvent structure factor $G_{\phi \phi}(r \rightarrow \infty) \propto \exp (-r / \xi) / r$. The profile $\bar{\phi}(z)$ is the analytic solution of the semiinfinite Ginzburg-Landau equation [41] obtained from minimizing $\Omega\left[\phi, \varrho_{ \pm}\right]$after expanding Eq. (1) up to fourth order in $\phi-\phi_{b}$ and neglecting the ion-solvent coupling, i.e., assuming $f_{ \pm}=0$, which implies $V_{ \pm}(\phi)=0$. Within Ginzburg-Landau theory the extrapolation length $z_{0}$ is fixed by the boundary condition $\bar{\phi}^{\prime}(0)=-3 h / \chi$. For $|h| \rightarrow \infty$ or $\xi \rightarrow \infty$ this leads to $|\bar{\phi}(0)| \rightarrow \infty$. However, within the context of the present study $\bar{\phi}(z)$ is an approximation of the volume fraction of solvent component $A$, which is restricted to the interval $[0,1]$. Hence, we accept the extrapolation length $z_{0}$ as determined from the boundary condition $\bar{\phi}^{\prime}(0)=-3 h / \chi$ only if this leads to $\bar{\phi}(0) \in[0,1]$. Otherwise the extrapolation length $z_{0}$ is inferred from the ersatz boundary condition $\bar{\phi}(0)=1$ if $h>0$ or $\bar{\phi}(0)=0$ if $h<0$.

The dashed line in Fig. 7(b) is the approximate electrostatic potential

$$
\begin{aligned}
& \bar{\psi}(z)=4 \operatorname{artanh}\left(C_{P B} \exp (-\kappa z)\right), \\
& C_{P B}:=\tanh \left(\frac{1}{2} \operatorname{arsinh}\left(\frac{2 \pi \ell_{B} \sigma}{\varepsilon\left(\phi_{b}\right) \kappa}\right)\right),
\end{aligned}
$$

which is the analytic solution of the semi-infinite PoissonBoltzmann equation [42] for a uniform permittivity $\varepsilon\left(\phi_{b}\right)$ and for neglecting the ion-solvent coupling (i.e., for $f_{ \pm}=$ $0)$.

Finally, the dashed lines in Figs. 7(c) and (d) are the approximate number density profiles of the \pm -ions,

$$
\bar{\varrho}_{ \pm}(z)=I_{b} \exp \left(-\left( \pm \bar{\psi}(z)+V_{ \pm}(\bar{\phi}(z))\right)+V_{ \pm}\left(\phi_{b}\right)\right)
$$

which correspond to the Boltzmann distributions of noninteracting particles in the external fields due to the approximate electrostatic potential $\bar{\psi}(z)$ and the approximate composition $\bar{\phi}(z) ; I_{b}=\varrho_{ \pm}(z \rightarrow \infty)$. Whereas the composition profile $\bar{\phi}(z)$ (Eq. (18)) and the electrostatic potential profile $\bar{\psi}(z)$ (Eq. (19)) are independent of the ion-solvent coupling $V_{ \pm}$, the ion number density profiles $\bar{\varrho}_{ \pm}(z)(\mathrm{Eq} \cdot(20))$ are not.

At distances from the wall of more than a few particle diameter $(z>2)$ the approximate profiles $\bar{\phi}(z), \bar{\psi}(z)$, and $\bar{\varrho}_{ \pm}(z)$ differ only slightly from the ones obtained by the full numerical minimization. Closer to the wall the deviations between the numerical and the approximate profiles are more pronounced, but in this spatial range the present local model is not conclusive because it neglects the surface layering of actual fluids. A similar situation occurs within BCA [19] (see Fig. 3(a) therein) shown in Fig. 8 . There, at distances $\widetilde{z}<2 \AA$, the solvent composition profile $\phi(\widetilde{z})$ for strong ion-solvent coupling ( $f_{+}=30, f_{-}=0$, solid line) differs strongly from that in the absence of ion-solvent coupling $\left(f_{+}=f_{-}=0\right.$, dashed line). At large distances the deviations are small. A closer comparison between Fig. 8 and Fig. 7(a) would require the knowledge of the particle size, which is however not specified in Ref. [19]. A description of the presently considered semi-infinite planar system within RPA has been given in Ref. [9]. There the ion-solvent coupling has been treated perturbatively, but the full, numerically determined profiles $\phi(z), \psi(z)$, and $\varrho_{ \pm}(z)$ within RPA 

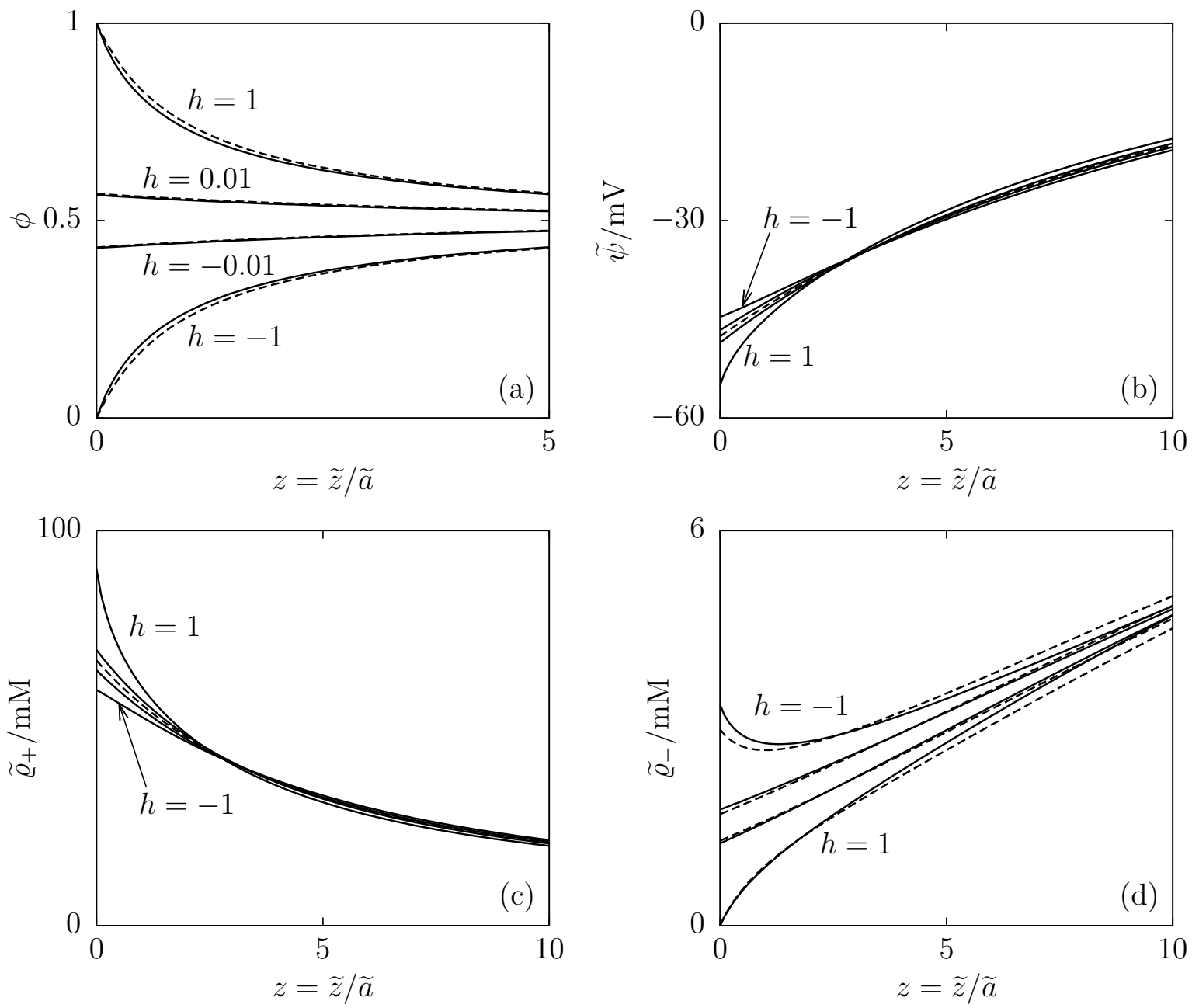

FIG. 7: Profiles of the volume fraction $\phi$ of solvent component $A[(\mathrm{a})]$, the electrostatic potential $\widetilde{\psi}($ with $\widetilde{\psi}(z \rightarrow \infty) \rightarrow 0)$ $[(\mathrm{b})]$, the cation number density $\widetilde{\varrho}_{+}[(\mathrm{c})]$, and the anion number density $\widetilde{\varrho}_{-}[(\mathrm{d})]$ in a semi-infinite system bounded by a wall at $z=\widetilde{z} / \widetilde{a}=0$ with surface charge density $\widetilde{\sigma}=-1 \mu \mathrm{C} / \mathrm{cm}^{2}$ and surface field strength $h$. These results correspond to Gibbs free energies of transfer $f_{+}=0, f_{-}=20$, the bulk volume fraction $\phi_{b}=0.5$ of solvent component $A$, and the bulk ionic strength $\widetilde{I}=\widetilde{\varrho}_{ \pm b}=10 \mathrm{mM}$. The Flory-Huggins parameter $\chi(T)$ is chosen to correspond to that temperature, for which the bulk correlation length $\xi$ is half of the Debye length $1 / \kappa$, which is taken to be temperature independent (see Fig. 6). For the specified surface fields $h$ the solid lines are the numeric solutions obtained from the density functional model in Eq. (1) within LDA. For reasons of clarity in (b) and (c) the full lines for $h= \pm 0.01$ are not designated; they can be nonetheless identified in an obvious way. The dashed lines correspond to the approximate profiles $\bar{\phi}(z), \bar{\psi}(z)$, and $\bar{\varrho}_{ \pm}(z)$ introduced in Eqs. (18), (19), and (20), respectively. Note that $\bar{\psi}(z)$ and, due to the choice $f_{+}=0, \bar{\varrho}_{+}(z)$ are independent of the magnitude $|h|$; therefore both in (b) and (c) there is only one dashed line. For $z>2$ the approximate profiles differ only slightly from the ones obtained by a full numerical minimization. Density oscillations close to the wall, which are expected in actual fluids, do not occur, because packing effects are not captured by the present square-gradient approach.

have not been discussed. However, by comparing these profiles, as obtained within RPA, with those obtained within BCA or LDA one could assess the influence of non-locality on the interfacial structure in the complex fluids studied here.

\section{B. Critical adsorption}

Here we investigate critical adsorption at a wall with a strong surface field $h$ and with surface charge density $\sigma$.
We consider the case that in the bulk the binary liquid mixture is at the critical bulk composition $\phi_{b}=\phi_{c}$ in the presence of salt with bulk ionic strength $\varrho_{ \pm b} \equiv I=I_{c}$ (see Subsec. III A). A surface field $h>0(h<0)$ favors the adsorption of $A(B)$ particles and leads to a local segregation. In order to obtain an analytical expression for the excess adsorption $\Gamma(\xi)$, which captures the full meanfield behavior to leading order close to the critical point $(\xi \rightarrow \infty)$, we expand the density functional in Eq. (1) in two steps in order to derive a Ginzburg-Landau-type description. In the first step the density functional $\Omega\left[\phi, \varrho_{ \pm}\right]$ 


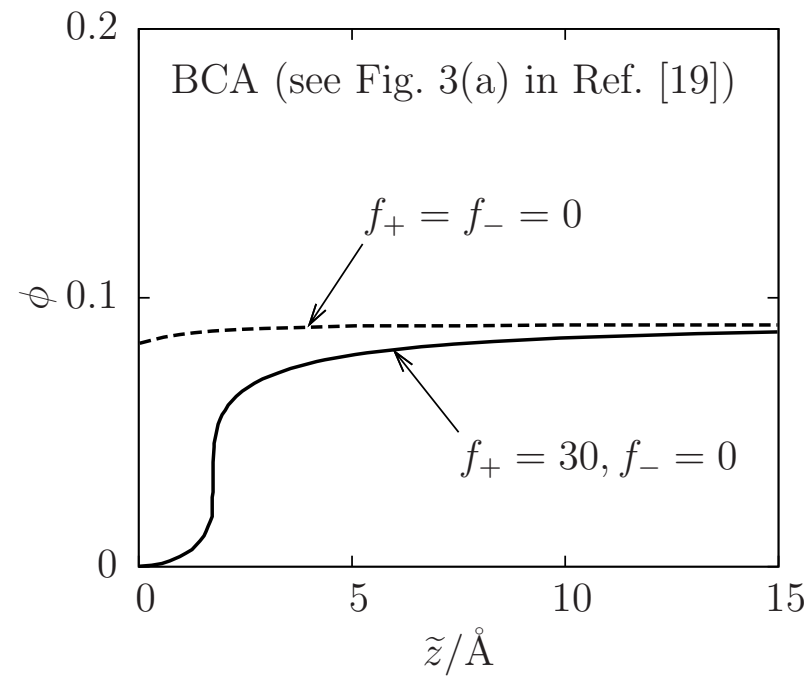

FIG. 8: Composition profiles $\phi(\widetilde{z})$ within BCA for strong ionsolvent coupling $\left(f_{+}=30, f_{-}=0\right.$, solid line) and in the absence of ion-solvent coupling $\left(f_{+}=f_{-}=0\right.$, dashed line) taken from Fig. 3(a) in Ref. [19]. Here a solvent with $\varepsilon_{A}=80$ and $\varepsilon_{B}=20$ at bulk composition $\phi_{b}=0.09$ is considered. The bulk ionic strength is $\widetilde{I}=0.1 \mathrm{mM}$ and the surface charge density is $\widetilde{\sigma}=-16 \mu \mathrm{C} / \mathrm{cm}^{2}$. At distances $\widetilde{z}<2 \AA$ from the wall the two curves differ strongly from each other, whereas the differences are small at large distances.

in Eq. (1) is expanded up to second order in the deviations $\Delta \varrho_{ \pm}(z):=\varrho_{ \pm}(z)-I$ of the ion densities from their bulk equilibrium values $I=I_{c}$. This leads to a density functional $\Omega_{1}\left[\phi, \Delta \varrho_{ \pm}\right]$. Minimizing $\Omega_{1}\left[\phi, \Delta \varrho_{ \pm}\right]$ with respect to $\Delta \varrho_{ \pm}$renders Euler-Lagrange equations linear in $\Delta \varrho_{ \pm}(z)$, the solutions of which are functionals $\Delta \varrho_{ \pm}^{*}(z,[\phi])$ of the (up to here unknown) solvent composition profile $\phi$. Inserting the solutions $\Delta \varrho_{ \pm}^{*}(z,[\phi])$ into the density functional, $\Omega_{1}\left[\phi, \Delta \varrho_{ \pm}\right]$and, as the second step, expanding $\Omega_{1}\left[\phi, \Delta \varrho_{ \pm}^{*}[\phi]\right]$ up to fourth order in the order parameter deviations $\varphi(z):=\phi(z)-\phi_{c}$ leads to the Ginzburg-Landau-type functional

$$
\begin{aligned}
\frac{\mathcal{H}[\varphi]}{A}= & \int_{0}^{\infty} \mathrm{d} z\left(a(\varphi(z))^{2}+b(\varphi(z))^{4}+c\left(\varphi^{\prime}(z)\right)^{2}\right. \\
& +U(z) \varphi(z))-h \varphi(0)+\mathcal{O}\left((\Delta \gamma)^{2}\right)
\end{aligned}
$$

where $A$ is the surface area in units of $\widetilde{a}^{2}$. Here $a=\chi_{c}-\chi$, $b=4 / 3, c=\chi / 6$, and the effective "external" field is

$$
U(z)=-\frac{\kappa \sigma \Delta \gamma}{2} \exp (-\kappa z)-\frac{2 \pi \ell_{B} \sigma^{2} \varepsilon^{\prime}\left(\phi_{c}\right)}{\left(\varepsilon\left(\phi_{c}\right)\right)^{2}} \exp (-2 \kappa z) .
$$

The external field $U(z)$ describes the influence of surface charges $\sigma$ on the order parameter $\varphi$. The first term on the right-hand side of Eq. (22) is due to the ion solubility whereas the second term is due to the dielectric properties of the solvent. Solving perturbatively to first order in $U$ the Euler-Lagrange equation, obtained from $\mathcal{H} / A$ in Eq. (21), leads to the equilibrium order parameter profile $\varphi_{\mathrm{eq}}(z ; \xi)$.
From Eq. (21) one obtains the bulk correlation length $\xi=\lambda+\mathcal{O}\left((\Delta \gamma)^{2}\right)=\lambda \sqrt{1-g^{2}}+\mathcal{O}\left((\Delta \gamma)^{2}\right)=\xi^{(O Z)}+$ $\mathcal{O}\left((\Delta \gamma)^{2}\right)$ with $\lambda=\sqrt{\frac{\chi}{6\left(\chi_{c}-\chi\right)}}=\sqrt{\frac{c}{a}}$ (see Eqs. (9), (10), and (12) with $\chi_{s}\left(\phi_{c}, I_{c}\right)=\chi_{c}$ ) for $\chi<\chi_{c}$. In the following we shall neglect the corrections $\mathcal{O}\left((\Delta \gamma)^{2}\right)$, which are expected to be small within LDA (see Sec. III). Using the empirical form $\chi(T)=\chi_{S}+\frac{\chi_{H}}{T}$ (see Sec. II) one obtains $\xi\left(T \rightarrow T_{c}\right) \stackrel{t \rightarrow 0}{\rightarrow} \xi_{0}^{+}|t|^{-\nu}$ with the nonuniversal critical amplitude $\xi_{0}^{+}=\sqrt{\frac{\chi_{c} T_{c}}{6\left|\chi_{H}\right|}}$, the critical (mean-field) exponent $\nu=1 / 2$, and $t=\left(T-T_{c}\right) / T_{c}$. Moreover, from Eq. (21) one obtains the bulk order parameter $\varphi_{\mathrm{eq}}(z=\infty ; \xi)=\sqrt{-\frac{a}{2 b}}=\sqrt{\frac{3\left(\chi-\chi_{c}\right)}{8}} \stackrel{t \rightarrow 0}{\rightarrow}$ $m_{0}|t|^{\beta}$ with the critical amplitude $m_{0}=\sqrt{\frac{3\left|\chi_{H}\right|}{8 T_{c}}}$ and the critical (mean-field) exponent $\beta=1 / 2$. For later purposes (see the text below Eqs. (23) and (26)) here we note that $\sqrt{c / b}=\sqrt{2 \chi_{c}} / 4=\sqrt{2} m_{0} \xi_{0}^{+}=1 / 2$ for $\chi=\chi_{c}=2$ (see Sec. III A).

At the critical point $(\xi=\infty)$ and far away from the substrate the equilibrium order parameter profile $\varphi_{\mathrm{eq}}(z ; \xi=\infty)$ decays as

$$
\begin{aligned}
\varphi_{\mathrm{eq}}(z & \rightarrow \infty ; \xi=\infty)=\frac{\operatorname{sign}(h)}{2 z} \\
& +\left(-\frac{\operatorname{sign}(h)}{2}+\frac{9 \sigma \Delta \gamma}{10 \kappa^{3}}+\frac{9 \pi \ell_{B} \sigma^{2} \varepsilon^{\prime}\left(\phi_{c}\right)}{40 \kappa^{4}\left(\varepsilon\left(\phi_{c}\right)\right)^{2}}\right) \frac{1}{z^{2}} \\
& +\mathcal{O}\left(z^{-3}\right) .
\end{aligned}
$$

The leading contribution $\operatorname{sign}(h) \frac{\sqrt{c / b}}{z}$ can be written in the scaling form $\operatorname{sign}(h) m_{0} c_{+}\left(z / \xi_{0}^{+}\right)^{-\beta / \nu}$ with the universal amplitude $c_{+}=\sqrt{2}$ [43], where the critical exponents take their mean-field values $\beta=\nu=\frac{1}{2}$ [33]. Accordingly, the leading term in Eq. (23) is not affected by the surface charge, the presence of ions, or the dielectric properties of the solvent. However, these materials properties do modify the amplitude of the subleading contribution $\left(\propto 1 / z^{2}\right)$.

Close to the critical point $(\xi \rightarrow \infty)$ the excess adsorption $\Gamma(\xi)=\int_{0}^{\infty} \mathrm{d} z \varphi_{\text {eq }}(z ; \xi)$ with the perturbatively obtained profile $\varphi_{\mathrm{eq}}(z ; \xi)$ (see above) is given by

$$
\Gamma(\xi)=\Gamma_{0}(\xi)+\Gamma_{1}(\xi)+\mathcal{O}(1 / \xi)
$$

with

$$
\Gamma_{0}(\xi):=\frac{\operatorname{sign}(h)}{2} \ln (2 \xi)
$$

and

$$
\Gamma_{1}(\xi):=\frac{3 \sigma \Delta \gamma}{8 \kappa^{2}}+\frac{3 \pi \ell_{B} \sigma^{2} \varepsilon^{\prime}\left(\phi_{c}\right)}{16 \kappa^{3}\left(\varepsilon\left(\phi_{c}\right)\right)^{2}}
$$




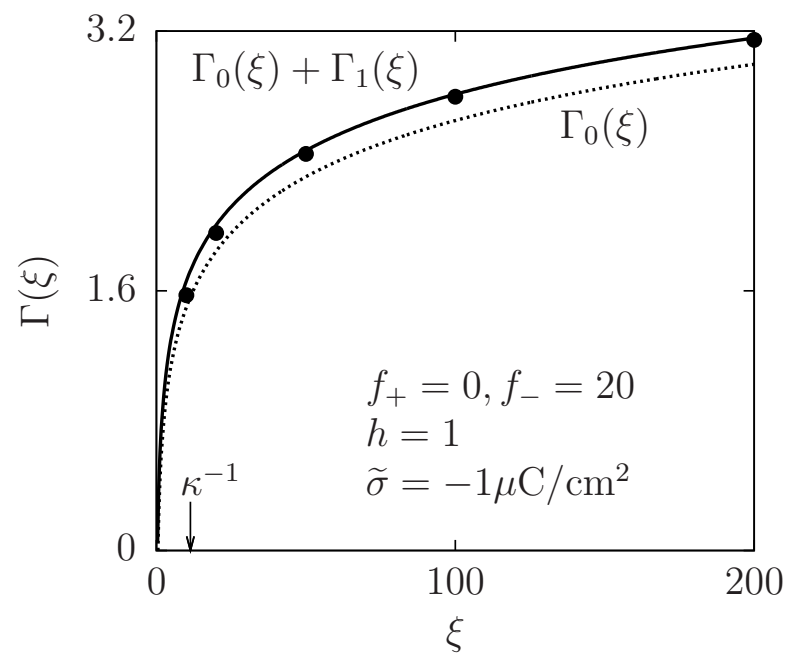

FIG. 9: Comparison of the numerically calculated excess adsorption $\Gamma(\xi)$ obtained within the full model in Eq. (1) (•) with the predictions of Eq. (24) for the parameters used in Fig. 7 with $h=1$. The Debye length $\kappa^{-1}$ (marked by an arrow) corresponds to a bulk ionic strength $\widetilde{I}=10 \mathrm{mM}$. The term $\Gamma_{0}(\xi)$ (dotted line, see Eq. (25)), which contains the leading contribution to the excess adsorption and which corresponds to a vanishing surface charge density $(\sigma=0)$, exhibits visible deviations from the numerical results $(\bullet)$; nonetheless $\Gamma_{1}(\xi) / \Gamma_{0}(\xi) \rightarrow 0$ for $\xi \rightarrow \infty$. Taking into account in addition the term $\Gamma_{1}(\xi)$ (see Eq. (26)), which exhibits a dependence on the surface charge $\sigma$, quantitative agreement is found between $\Gamma_{0}(\xi)+\Gamma_{1}(\xi)$ (solid line) and the numerical results $(\bullet)$ in the limit $\xi \rightarrow \infty$. This finding also implies that those terms of Eq. (1), which have been left out upon deriving Eq. (21), do not contribute detectably.

The leading contribution $\operatorname{sign}(h) \sqrt{\frac{c}{b}} \ln (\xi)$ can be written in the scaling form $\simeq \operatorname{sign}(h) m_{0} \xi_{0}^{+} g_{+}(-\ln (|t|))$ with the universal amplitude $g_{+}=\sqrt{2} \nu=1 / \sqrt{2}$ within meanfield theory [43]. $\Gamma_{0}(\xi)$ diverges for $\xi \rightarrow \infty$ whereas $\Gamma_{1}(\xi)$ remains finite and thus represents the first subdominant correction. Figure 9 compares the predictions of Eq. (24) with the results obtained by numerically calculating the excess adsorption within the full model as given by Eq. (1) (•) for the parameters used in Fig. 7 with $h=1$, in particular for large $\xi$. Whereas the leading contribution $\Gamma_{0}(\xi)$ in Eq. (25) itself (dotted line) deviates visibly from the full numerical results $(\bullet)$, there is quantitative agreement between the latter and $\Gamma_{0}(\xi)+\Gamma_{1}(\xi)$ (solid line) in the limit $\xi \rightarrow \infty$. Since $\Gamma_{0}(\xi)$ corresponds to a vanishing surface charge $(\sigma=0)$, the difference between the dotted and the full line in Fig. 9 demonstrates the influence of electrostatic interactions on the excess adsorption. The quantitative agreement of $\Gamma_{0}(\xi)+\Gamma_{1}(\xi)$ (solid line) with the numerical results $(\bullet)$ indicates that the terms of Eq. (1) neglected upon deriving Eq. (21) do not contribute detectably to the leading and the first subleading behavior of $\Gamma(\xi \rightarrow \infty)$. Moreover, for the given choice of parameters the magnitude of the correc- tion $|\mathcal{O}(1 / \xi)|$ in Eq. (24) turns out to be smaller than $\left|\Gamma_{1}(\xi)\right|$, which in turn vanishes relative to $\Gamma(\xi \rightarrow \infty)$. Note that within BCA, for $f_{+}=0, f_{-}=20$ (as used in Fig. 9) the uniform bulk state is unstable in a certain vicinity of the critical point, because $|g|=1.39>1$ (see Sec. III B), which precludes calculating the excess adsorption $\Gamma(\xi)$.

Critical adsorption occurs upon approaching the critical point $(\xi \rightarrow \infty)$, where the excess adsorption diverges as $\Gamma \propto \ln \xi$, which is in agreement with the expected universal scaling behavior $\Gamma \propto \xi^{1-\beta / \nu}[43,44]$ for the classical exponents $\beta=\nu=1 / 2$ corresponding to the present mean-field theory. It is apparent from Eq. (24) that the leading contribution $\Gamma_{0}$ is not altered by the surface charge, the presence of ions, or the dielectric properties of the solvent. However, these non-universal properties do influence the subleading contribution $\Gamma_{1}$.

Recently the adsorption of critical water+2,6dimethylpyridine mixtures with $\mathrm{KBr}$ of various ionic strengths $I$ has been investigated by means of surface plasmon resonance [34]. For the case of a hydrophobic wall the excess adsorption turned out to be practically independent of the ionic strength. This is in agreement with Eq. (24) because a hydrophobic wall is only weakly charged [45] such that the second and third terms on the right-hand side of Eq. (24) are negligibly small.

For the case of a hydrophilic, negatively charged $(\sigma<$ 0 ) wall a decrease of the adsorption of water has been measured upon adding salt [34]. Hydrophilic walls can be expected to be strongly charged [46] such that $\sigma=$ $\operatorname{sign}(\sigma) \sigma_{\text {sat }}$ with the saturation surface charge density $\sigma_{\text {sat }}=\kappa \varepsilon\left(\phi_{c}\right) /\left(\pi \ell_{B}\right)[47]$. In this case from Eq. (24) one obtains

$$
\frac{\partial \Gamma}{\partial I}=-\frac{3}{4 \kappa^{3}}\left(2 \operatorname{sign}(\sigma) \Delta \gamma+\frac{\varepsilon^{\prime}\left(\phi_{c}\right)}{\varepsilon\left(\phi_{c}\right)}\right) .
$$

Equation (27) assumes only a weak dependence of $\phi_{c}$ (and thus of $\Delta \gamma$ and of $\varepsilon\left(\phi_{c}\right)$ ) on the ionic strength $I$ (see Sec. III A) so that the derivative $\frac{\mathrm{d} \phi_{c}}{\mathrm{~d} I}$ does not appear.

If 2,6-dimethylpyridine is denoted as the $A$ component and water as the $B$ component of the binary liquid mixture (i.e., $\Gamma$ measures the excess of 2,6-dimethylpyridine), at the lower critical demixing point an experimental value of $\varepsilon^{\prime} / \varepsilon \approx-1.2$ is found $[48,49]$. For this mixture the solubility contrasts for $\mathrm{KBr}$ are $f_{+} \approx 2.5$ and $f_{-} \approx 8.4$ [24] which leads to $f_{+}-f_{-} \approx-5.9$ and $\Delta \gamma \approx-0.30$. Within LDA from these numbers one finds $\partial \Gamma / \partial I>0$ (i.e., decreasing water adsorption upon adding salt), and the second (dielectric) contribution on the right-hand side of Eq. (27) dominates. In contrast, within BCA one has $\partial \Gamma^{(\mathrm{BCA})} / \partial I<0$ (i.e., increasing water adsorption upon adding salt), because $\Delta \gamma \approx f_{+}-f_{-}$leads to a dominance of the first (ion solubility) contribution on the right-hand side of Eq. (27). Hence the overestimation of the ion-solvent coupling within BCA leads to a sign of $\partial \Gamma^{(\mathrm{BCA})} / \partial I$ which is not compatible with the aforementioned experimental findings in Ref. [34], whereas the sign 
of $\partial \Gamma / \partial I$ within the present LDA is in agreement with these findings. Since the dielectric properties are experimentally accessible one could use Eq. (27) to determine $\Delta \gamma$ from measurements of the excess adsorption $\Gamma$ as a function of the ionic strength $I$. A comparison of this resulting value for $\Delta \gamma$ with the difference $f_{+}-f_{-}$of the Gibbs free energies of transfer (inferred, e.g., from electrochemical methods) would be a direct way to probe quantitatively the difference between the LDA and the BCA.

In order to further test the different predictions following from BCA and LDA we suggest additional adsorption measurements for hydrophilic walls. For $\mathrm{KBr}$ as salt the arguments above lead to the assertions that, within LDA, one has $\partial \Gamma / \partial I>0$ (i.e., decreasing water adsorption upon adding salt) independently of the sign of the surface charge $\sigma$ (because the last term on the righthand side of Eq. (27) dominates), whereas within BCA $\partial \Gamma^{(\mathrm{BCA})} / \partial I$ is expected to change sign upon changing the sign of $\sigma$ (because the first term on the right-hand side of Eq. (27) dominates). More interestingly, using an antagonistic electrolyte (i.e., with $f_{+}$and $f_{-}$having opposite signs $)$ such as $\operatorname{HBr}\left(f_{+} \approx-11.2, f_{-} \approx 8.4\right.$ [24], i.e., $\Delta \gamma \approx-4$ ) the first (ion solubility) contribution on the right-hand side of Eq. (27) is dominating such that $\partial \Gamma / \partial I$ and $\sigma$ are expected to have the same sign. In this case, upon adding salt, the amount of adsorbed water either decreases or increases depending on the sign of the surface charge. This is in contrast to electrowetting where the water adsorption increases with the magnitude but independent of the sign of the surface charge [50]. Within the BCA approach of Ref. [21] the difference for cations and anions with respect to their solubility contrasts in the two pure solvent components is neglected (i.e., $f_{+}=f_{-} \gg 1$ so that $\Delta \gamma=0$ ) to the effect that the reported capillary condensation-like adsorption of water between two equally charged walls at variable distance should be independent of the sign of the surface charge. The analysis above implies that the same property is expected to occur for sufficiently small values of $|\Delta \gamma|$, but the adsorption may depend on the sign of the surface charge if $|\Delta \gamma|$ becomes of the order unity.

\section{COLLOIDAL INTERACTIONS IN NEAR-CRITICAL ELECTROLYTE SOLUTIONS}

The interactions between colloidal particles in a fluid medium comprise dispersion forces, direct screened Coulomb forces, steric forces, as well as solvent-mediated interactions, which are commonly referred to as solvation forces. If the thermodynamic state of the fluid medium is moved towards a critical point, e.g., the critical demixing point of a binary liquid mixture, the fluctuation induced long-ranged, and universal critical Casimir force emerges; this singular contribution to the solvation force dominates [51-53]. In the presence of a sufficiently strong adsorption preference of colloids or of confining walls for one of the components of the solvent the critical Casimir force depends only on the relative signs of the surface fields $h$ acting on the order parameter at these surfaces (and on the geometry of the latter) but not on non-universal material parameters. The critical Casimir force is attractive (repulsive) if the surface fields have equal (opposite) signs.

While being investigated theoretically for quite some time [51], the direct experimental verification of the critical Casimir effect has been achieved only recently for a single colloidal particle dispersed in a mixture of water and 2,6-dimethylpyridine close to a planar wall [53-55]. In that study the measured effective colloid-wall interaction potential was interpreted in terms of a superposition of a repulsive screened Coulomb force and the critical Casimir force. Within this picture the direct electrostatic repulsion dominates for temperatures $T$ which deviate from $T_{c}$ more than a few tenth of a Kelvin, whereas an increasingly strong Casimir attraction (repulsion) occurs for symmetric (antisymmetric) boundary conditions upon approaching the critical point $\left(T \rightarrow T_{c}\right)$. Recently these experiments have been modeled within RPA, which provides a satisfactory fitting of the experimental curves in Refs. [54, 55]. However, this approach involves a large number of model parameters [56], which limits the conclusiveness of these fits.

The influence of adding salt onto the effective colloidwall interaction has been studied recently [34] using the same experimental setup as in Refs. [54, 55]. It turns out that with $10 \mathrm{mM}$ of $\mathrm{KBr}$ the Casimir attraction for symmetric boundary conditions starts to dominate the direct electrostatic repulsion already several Kelvin away from the critical point (instead of tenths of a Kelvin as for the salt-free solvent). Moreover, for antisymmetric boundary conditions, for which both the direct electrostatic and the critical Casimir forces are expected to be repulsive, in the presence of salt an attraction has been detected within an intermediate temperature range. The latter observation demonstrates that, under certain conditions, assuming the simple superposition of the direct electrostatic and the critical Casimir forces is insufficient to understand the actual effective interaction.

Based on the Ginzburg-Landau-like description in Eq. (21), which follows from the full model given in Eq. (1), we have identified a mechanism giving rise to the aforementioned unexpected attraction for antisymmetric boundary conditions in the presence of salt [26]. For $\Delta \gamma \neq 0$ the cations and anions are separated close to the surfaces, where the order parameter $\varphi_{\text {eq }}$ is non-uniform. This gives rise to dipolar layers, which can interact with the surface charges at the distant surface. These dipole layers are expected to contribute significantly to the effective interaction if the direct electrostatic interaction is weak; this is the case for antisymmetric boundary conditions, for which the hydrophobic wall is expected to be weakly charged. If the direct electrostatic interaction is strong, which is expected to occur for symmetric boundary conditions with hydrophilic surfaces, the dipo- 
lar layers do not significantly contribute to the effective interaction. In that case the sole effect of adding salt is to reduce the Debye length and thereby to weaken the direct electrostatic repulsion relative to the critical Casimir force. Therefore, with salt the onset of effective attraction occurs at temperatures $T$ further away from $T_{c}$ than in the salt-free case [26].

A conceivable alternative mechanism for the emergence of an effective attraction in the case of antisymmetric boundary conditions has been proposed which is independent of differences in the solubility of cations and anions [57]. It has been argued within RPA that a charged wall (of either polarity) accumulates an increased number of ions compared to an uncharged wall. Due to this enhanced total density of ions (which are hydrophilic independent of their sign) a charged wall should, from a distance, appear increasingly hydrophilic upon adding salt such that for certain system parameters an underlying actually hydrophobic character of a wall might be overcompensated and turn into an effectively hydrophilic wall; actually hydrophilic walls remain so upon adding salt [57]. Such a salt-induced apparent hydrophilicity, which would occur on the surfaces of all dissolved colloids, would in turn lead to effectively symmetric boundary conditions and thus to attractive solvation forces. However, according to Fig. 9, even in the presence of salt the excess adsorption follows the actual preference of the surface field, also upon approaching $T_{c}$. As mentioned in Sec. IV B, it is not possible to calculate the excess adsorption within BCA (and RPA) for the parameters used in Fig. 9, because for them within this approach the uniform bulk state is thermodynamically unstable. Thus Fig. 9 demonstrates that within LDA salt-induced apparent hydrophilicity does not occur (i.e., $\Gamma$ does not become negative). Therefore there is reason to expect that salt-induced apparent hydrophilicity is an artifact of the BCA and the RPA. In addition, by means of surface plasmon resonance it has been checked experimentally that the adsorption preference, in particularly of hydrophobic substrates, is not altered by adding salt [34]. Therefore, for antisymmetric boundary conditions of the order parameter, there are doubts that salt-induced apparent hydrophilicity can serve as an explanation for the experimentally observed effective attraction within an intermediate temperature range.

Recently, additional numerical studies within BCA have been performed suggesting that ion-induced "precipitation" [8] or non-linearities [58] influence the effective colloid-colloid interaction. However, our results in Secs. III and IV concerning the reliability of BCA point towards the possibility that those proposed effects are artifacts of the BCA due to an overestimation of the ion-solvent coupling. Therefore it would be worthwhile to reconsider the aforementioned proposed effects within LDA, which has been shown to be consistent with presently available experimental evidence.

\section{CONCLUSIONS AND SUMMARY}

We have derived a local density approximation (LDA) for the density functional of point-like ions interacting locally with a binary liquid mixture acting as a solvent (Fig. 2), which for large free energies of transfer of the ions improves the frequently used bilinear coupling approximation (BCA). It turns out that within the proposed LDA, and in contrast to the BCA, the influence of ions on the bulk phase diagram (Fig. 1), the critical point (Fig. 3), and the bulk structure (Figs. 4-6) is predicted to be weak. This is in agreement with the presently available experimental data. The interfacial structure at distances from a charged wall less than a few particle diameters can be predicted by neither BCA nor LDA, because none of these two local models accounts for the layering due to packing effects which dominate in actual fluids at such short distances. But, within both BCA and LDA, further away from the wall the system can be described reliably in simple terms of a uniform permittivity and an effective surface field. This description nonetheless captures the actual structure as obtained from the full numerical minimization (Fig. 7). Upon approaching the critical point the subleading (but not the leading) contribution to critical adsorption is found to be sensitive to system and materials parameters such as the bulk ionic strength, solubility properties, surface charges, and the permittivities of the solvent components (Fig. 9).

If a salt disturbs the molecular arrangement of the solvent molecules only at small distances, it is modeled here by point-like ions which interact locally with the solvent. Such a salt does neither significantly alter the bulk phase behavior nor the bulk structure or the asymptotic decay of density profiles at walls. But it contributes to the interfacial structures up to distances of the order of the Debye length $1 / \kappa$ as well as to critical adsorption in subleading order. If the solvent is moved thermodynamically towards a critical point, where the bulk correlation length $\xi$ diverges, the ratio $1 /(\kappa \xi)$ of the range $1 / \kappa$ of the ionrelated surface structure and $\xi$ becomes small, such that the leading, universal critical behavior of the solvent is not altered by adding salt[26].

Whether the description of a given electrolyte solution within a local model is justified or not does not depend on the salt alone but on the combination of salt and solvent. Experimentally observed effects in binary liquid mixtures due to adding salt, such as the shift of the critical point, depend sensitively on the type of mixture (compare Ref. [31] for water+2,6-dimethylpyridine and Ref. [14] for heavy water+3-methylpyridine). Moreover, the measured critical point shifts exhibit a strong dependence on the size of the ions (compare Ref. [14] for alkali halides and Ref. [15] for sodium tetraphenylborate). These evidences in combination with the present analysis, which implies a weak influence of the ionic charge, lead to the conclusion that steric effects might play an important role for the ion-solvent interaction. This interpretation is supported by reports of critical point shifts of similar 
magnitude in binary liquid mixtures due to adding nonionic impurities [59]. Consequently it appears as if it is mostly the property of an ion to be a structure maker or a structure breaker and only to a lesser extent its electric charge which determines the influence of a salt onto the properties of the solvent. In order to obtain quantitatively reliable predictions for electrolyte solutions, a larger effort has to be devoted to study the steric and chemical influence of ions on the solvent.

In summary, for ions dissolved in a binary solvent the bulk phase diagrams (Fig. 1), the critical point shifts (Fig. 3), the bulk two-point correlation functions (Figs. 4-6), the surface structures (Fig. 7), and critical adsorption (Fig. 9) have been investigated within a novel local density approximation (LDA) for the ion-solvent interaction (Fig. 2). The commonly used bilinear coupling approximation (BCA) turns out to strongly overestimate the influence of ions on the solvent properties in cases of realistic solvation free energies, whereas the LDA introduced here, in agreement with various available experimental data, predicts small effects. Although the presented LDA is expected to be more accurate than the $\mathrm{BCA}$, the former requires the same and not more parameters than the latter. According to its derivation, the LDA is expected to be reliable for small ionic strengths and on length scales larger than the particle size. Both available and possible future experiments have been discussed to probe and to explore the reliability and the range of validity of the present theoretical approach.

\section{Acknowledgments}

We thank M. Oettel, U. Nellen, J. Dietrich, and C. Bechinger for many stimulating discussions. A.G. is supported by MIUR within "Incentivazione alla mobilità di studiosi stranieri e italiani residenti all'estero."

\section{Appendix A: Derivation of the solvent-induced ion potential $V_{ \pm}(\phi)$}

Within the present model the solvation of ions is dominated by short-ranged interactions with solvent particles. Accordingly, the ion-solvent interaction is modeled locally in terms of a free energy density $\sum_{i= \pm} \varrho_{i} V_{i}(\phi)$ (see Eq. (1)). In order to derive an expression for the solvent-induced ion potential $V_{ \pm}(\phi)$, resorting to a lattice gas model and in line with the short range of the ion-solvent interaction, a single site is considered which is occupied by one solvent particle (either of type $A$ or of type $B$, i.e., corresponding to occupation numbers $\left.N_{A}=1-N_{B} \in\{0,1\}\right)$ and an arbitrary number of positive and negative ions $\left(N_{ \pm} \in \mathbb{N}_{0}\right)$. The surrounding of this considered site acts as a particle reservoir which is described by chemical potentials $\lambda_{i} k_{B} T, i \in\{A, B,+,-\}$, and the interaction energy per $k_{B} T$ of two particles of species $i, j \in\{A, B,+,-\}$ on that site is given by $k_{i j}$ with $k_{i j}=0$ for $i, j \in\{A, B\}$, because a particle does not interact with itself and the site cannot be occupied by more than one particle of type $A$ or $B$. The chemical potentials $\lambda_{i}$ are related to the chemical potentials $\mu_{\phi}$ and $\mu_{ \pm}$introduced in Sec. II (see below). Accordingly, the Hamiltonian $H k_{B} T$ of a configuration $\left(N_{A}, N_{B}, N_{+}, N_{-}\right) \in\{0,1\} \times\{0,1\} \times \mathbb{N}_{0} \times \mathbb{N}_{0}$ on one site with $N_{A}+N_{B}=1$ is given by

$$
\begin{aligned}
H\left(N_{A}, N_{B}, N_{+}, N_{-}\right)= & k_{A+} N_{A} N_{+}+k_{A-} N_{A} N_{-}+ \\
& k_{B+} N_{B} N_{+}+k_{B-} N_{B} N_{-}+ \\
& H_{\text {ion }}\left(N_{+}, N_{-}\right)
\end{aligned}
$$

with the ionic part

$$
\begin{aligned}
& H_{\text {ion }}\left(N_{+}, N_{-}\right)= \\
& k_{++} \frac{N_{+}\left(N_{+}-1\right)}{2}+k_{+-} N_{+} N_{-}+k_{--} \frac{N_{-}\left(N_{-}-1\right)}{2} .
\end{aligned}
$$

The corresponding grand partition function is

$$
\begin{array}{r}
\zeta\left(\lambda_{A}, \lambda_{B}, \lambda_{+}, \lambda_{-}\right)=\sum_{\left(N_{A}, N_{B}, N_{+}, N_{-}\right)} \\
\quad \exp \left(\sum_{i \in\{A, B,+,-\}} \lambda_{i} N_{i}-H\left(N_{A}, N_{B}, N_{+}, N_{-}\right)\right),
\end{array}
$$

where the outermost summation is over all configurations $\left(N_{A}, N_{B}, N_{+}, N_{-}\right) \in\{0,1\} \times\{0,1\} \times \mathbb{N}_{0} \times \mathbb{N}_{0}$ with $N_{A}+$ $N_{B}=1$. With $N_{A}=N_{\phi}, N_{B}=1-N_{\phi}$ for $N_{\phi} \in\{0,1\}$ the Hamiltonian in Eq. (A1) can be rewritten as

$$
\begin{aligned}
H\left(N_{\phi}, 1-N_{\phi}, N_{+}, N_{-}\right)= & f_{+} N_{\phi} N_{+}+f_{-} N_{\phi} N_{-}+ \\
& k_{B+} N_{+}+k_{B-} N_{-}+ \\
& H_{\text {ion }}\left(N_{+}, N_{-}\right)
\end{aligned}
$$

with the solvation energy difference $f_{ \pm}=k_{A \pm}-k_{B \pm}$ of a \pm -ion. The grand partition function in Eq. (A3) is $\zeta\left(\lambda_{A}, \lambda_{B}, \lambda_{+}, \lambda_{-}\right)=\exp \left(\lambda_{B}\right) Z\left(\mu_{\phi}, \mu_{+}, \mu_{-}\right)$with

$$
\begin{gathered}
Z\left(\mu_{\phi}, \mu_{+}, \mu_{-}\right)=\sum_{\left(N_{\phi}, N_{+}, N_{-}\right)} \exp \left(\sum_{i \in\{\phi,+,-\}} \mu_{i} N_{i}\right. \\
\left.-f_{+} N_{\phi} N_{+}-f_{-} N_{\phi} N_{-}-H_{\text {ion }}\left(N_{+}, N_{-}\right)\right),(\text {A } 5)
\end{gathered}
$$

where $\mu_{\phi}=\lambda_{A}-\lambda_{B}$ and $\mu_{ \pm}=\lambda_{ \pm}-k_{B \pm}$ (see Sec. II) and where the outermost summation is over all configurations $\left(N_{\phi}, N_{+}, N_{-}\right) \in\{0,1\} \times \mathbb{N}_{0} \times \mathbb{N}_{0}$.

In the limit of low ionic strength $\left(\mu_{ \pm} \rightarrow-\infty\right.$; this implies $\lambda_{ \pm} \rightarrow-\infty$ which in turn means that the averages $\left\langle N_{ \pm}\right\rangle=\varrho_{ \pm}$are small) one obtains

$$
\begin{aligned}
Z\left(\mu_{\phi}, \mu_{+}, \mu_{-}\right) \simeq & 1+\exp \left(\mu_{\phi}\right)+ \\
& \exp \left(\mu_{+}\right)\left(1+\exp \left(\mu_{\phi}-f_{+}\right)\right)+ \\
& \exp \left(\mu_{-}\right)\left(1+\exp \left(\mu_{\phi}-f_{-}\right)\right) .
\end{aligned}
$$

Using $\phi=\left\langle N_{\phi}\right\rangle=\frac{\partial \ln Z}{\partial \mu_{\phi}}$ and $\varrho_{ \pm}=\left\langle N_{ \pm}\right\rangle=$ $\frac{\partial \ln Z}{\partial \mu_{ \pm}}$one finds from the grand canonical potential 
$-k_{B} T \ln Z\left(\mu_{\phi}, \mu_{+}, \mu_{-}\right)$the Helmholtz free energy per $k_{B} T$

$$
\begin{aligned}
& -\ln Z\left(\mu_{\phi}, \mu_{+}, \mu_{-}\right)+\phi \mu_{\phi}+\varrho_{+} \mu_{+}+\varrho_{-} \mu_{-} \\
\simeq & \phi \ln \phi+(1-\phi) \ln (1-\phi)+ \\
& \varrho_{+}\left(\ln \varrho_{+}-1+\ln M_{+}\right)+\varrho_{-}\left(\ln \varrho_{-}-1+\ln M_{+}\right)
\end{aligned}
$$

with $M_{ \pm}=\left(1-\phi\left(1-\exp \left(-f_{ \pm}\right)\right)\right)^{-1}$. From Eq. (A7) one infers the effective ion-solvent interaction $V_{ \pm}(\phi):=$ $\ln M_{ \pm}$.
[1] S. Arrhenius, Z. phys. Chem. 1, 631 (1887).

[2] P. Debye and E. Hückel, Phys. Z. 24, 185 (1923).

[3] D.A. McQuarrie, Statistical Mechanics (University Science Books, Sausalito, 2000).

[4] W.B. Russel, D.A. Saville, and W.R. Schowalter, Colloidal dispersions (Cambridge University Press, 1989).

[5] A. Onuki and H. Kitamura, J. Chem. Phys. 121, 3143 (2004).

[6] A. Onuki, Phys. Rev. E 73, 021506 (2006).

[7] R. Okamoto and A. Onuki, Phys. Rev. E 82, 051501 (2010).

[8] R. Okamoto and A. Onuki, Phys. Rev. E 84, 051401 (2011).

[9] A. Ciach and A. Maciołek, Phys. Rev. E 81, 041127 (2010).

[10] V.M. Nabutovskii, N.A. Nemov, and Y.G. Peisakhovich, Phys. Lett. A 79, 98 (1980).

[11] V.M. Nabutovskii, N.A. Nemov, and Y.G. Peisakhovich, Mol. Phys. 54, 979 (1985).

[12] V.M. Nabutovskii and N.A. Nemov, J. Colloid Interface Sci. 114, 208 (1986).

[13] K. Sadakane, H. Seto, and M. Nagao, Chem. Phys. Lett. 426, 61 (2006).

[14] K. Sadakane, H. Seto, H. Endo, and M. Kojima, J. Appl. Cryst. 40, s527 (2007).

[15] K. Sadakane, H. Seto, H. Endo, and M. Shibayama, J. Phys. Soc. Jpn. 76, 113602 (2007).

[16] K. Sadakane, A. Onuki, K. Nishida, S. Koizumi, and H. Seto, Phys. Rev. Lett. 103, 167803 (2009).

[17] K. Sadakane, N. Iguchi, M. Nagao, H. Endo, Y.B. Melnichenko, and H. Seto, Soft Matter 7, 1334 (2011).

[18] Y. Tsori and L. Leibler, Proc. Natl. Acad. Sci. 104, 7348 (2007).

[19] D. Ben-Yaakov, D. Andelman, D. Harries, and R. Podgornik, J. Phys. Chem. B 113, 6001 (2009).

[20] A. Oleksy and J.-P. Hansen, Mol. Phys. 107, 2609 (2009).

[21] S. Samin and Y. Tsori, EPL 95, 36002 (2011).

[22] J.-P. Hansen and I.R. McDonald, Theory of simple liquids (Academic, London, 1986).

[23] Y. Marcus, Pure Appl. Chem. 55, 977 (1983).

[24] H.D. Inerowicz, W. Li, and I. Persson, J. Chem. Soc. Faraday Trans. 90, 2223 (1994).

[25] See the remark in Subsec. IIB of Ref. [6] that BCA "should not be taken too seriously".

[26] M. Bier, A. Gambassi, M. Oettel, and S. Dietrich, EPL 95, 60001 (2011).

[27] M. Rubinstein and R.H. Colby, Polymer physics (Oxford University Press, 2004).

[28] J.W. Cahn and J.E. Hillard, J. Chem. Phys. 28, 258 (1958).

[29] C.J.F. Böttcher, Theory of electric polarization (Elsevier, Amsterdam, 1973).

[30] R. Evans, Adv. Phys. 28, 143 (1979).
[31] C.Y. Seah, C.A. Grattoni, and R.A. Dawe, Fluid Phase Equilib. 89, 345 (1993).

[32] E.L. Eckfeldt and W.W. Lucasse, J. Phys. Chem. 47, 164 (1943).

[33] A. Pelissetto and E. Vicari, Phys. Rep. 368, 549 (2002).

[34] U. Nellen, J. Dietrich, L. Helden, S. Chodankar, K. Nygård, J.F. van der Veen, and C. Bechinger, Soft Matter 7, 5360 (2011).

[35] R. Evans, J.R. Henderson, D.C. Hoyle, A.O. Parry, and Z.A. Sabeur, Mol. Phys. 80, 755 (1993)

[36] R. Evans, R.J.F. Leote de Carvalho, J.R. Henderson, and D.C. Hoyle, J. Chem. Phys. 100, 591 (1994).

[37] R.J.F. Leote de Carvalho and R. Evans, Mol. Phys. 83, 619 (1994).

[38] J.G. Kirkwood, Chem. Rev. 19, 275 (1936).

[39] S. Dietrich and M. Napiórkowski, Phys. Rev. A 43, 1861 (1991).

[40] J.A. Davis, R.O. James, and J.O. Leckie, J. Colloid Interface Sci. 63, 480 (1978).

[41] T.C. Lubensky and M. Rubin, Phys. Rev. B 12, 3885 (1975).

[42] M. Gouy, J. de Physique 9, 457 (1910).

[43] G. Flöter and S. Dietrich, Z. Physik B 97, 213 (1995).

[44] S. Dietrich, in Phase transitions and critical phenomena, Vol. 12, edited by C. Domb and J.L. Lebowitz (Academic, London, 1988), p. 1.

[45] D. Rudhardt, C. Bechinger, and P. Leiderer, Prog. Colloid Polym. Sci. 110, 37 (1998).

[46] S.H. Behrens and D.G. Grier, J. Chem. Phys. 115, 6716 (2001).

[47] L. Bocquet, E. Trizac, and M. Aubouy, J. Chem. Phys. 117, 8138 (2002).

[48] D.R. Lide, Handbook of Chemistry and Physics, 79th ed. (CRC Press, Boca Raton, 1998).

[49] U. Kaatze and D. Woermann, J. Phys. Chem. 88, 284 (1984).

[50] F. Mugele and J.-Ch. Baret, J. Phys.: Condens. Matter 17, R705 (2005).

[51] M. Krech, The Casimir effect in critical systems (World Scientific, Singapore, 1994).

[52] F. Schlesener, A. Hanke, and S. Dietrich, J. Stat. Phys. 110, 981 (2003).

[53] A. Gambassi, J. Phys.: Conf. Ser. 161, 012037 (2009).

[54] C. Hertlein, L. Helden, A. Gambassi, S. Dietrich, and C. Bechinger, Nature 451, 172 (2008).

[55] A. Gambassi, A. Maciołek, C. Hertlein, U. Nellen, L. Helden, C. Bechinger, and S. Dietrich, Phys. Rev. E 80, 061143 (2009).

[56] F. Pousaneh, A. Ciach, and A. Maciołek, Soft Matter 8, 3567 (2012).

[57] F. Pousaneh and A. Ciach, J. Phys.: Condens. Matter 23, 412101 (2011).

[58] S. Samin and Y. Tsori, J. Chem. Phys. 136, 154908 
(2012).

[59] B.J. Hales, G.L. Bertrand, and L.G. Hepler, J. Phys.
Chem. 70, 3970 (1966). 